11

\title{
Соотношения взаимности для открытых нелинейных систем в переменных полях
}

\author{
(C) В.К. Игнатьев \\ Волгоградский государственный университет, \\ 400062 Волгоград, Россия \\ e-mail: vkignatjev@yandex.ru
}

Поступило в Редакцию 28 апреля 2021 г.

В окончательной редакции 6 сентября 2021 г.

Принято к публикации 24 сентября 2021 г.

В приближении марковской релаксации и локально квазиравновесного распределения методом Кубо получено доказательство соотношений взаимности для нелинейных систем в неоднородных переменных электрическом и магнитном полях в присутствии нестационарных спиновых токов, термодинамических потоков и механических возмущений.

Ключевые слова: нелинейные распределенные системы, локально квазиравновесное распределение, марковская релаксация, спиновый ток, взаимность.

DOI: 10.21883/JTF.2022.01.51861.126-21

\section{Введение}

Классические соотношения Онзагера симметрии кинетических коэффициентов [1] получены в линейном приближении, исходя из инвариантности макроскопического движения относительно обращения времени и предположения о том, что средняя релаксация спонтанных флуктуаций в системе происходит в соответствии с макроскопическими законами. Первоначально они применялись к термодинамическим процессам, сопровождающимся производством энтропии. Линейная связь между потоками и градиентами вводилась феноменологически. Квантовое обоснование соотношений взаимности, принадлежащее Кубо [2], выполнено с использованием равновесного оператора плотности, т.е. в линейном приближении, и не предполагает наличия в системе градиентов температуры и концентрации, а также потоков теплоты и вещества. Попытки применить метод Кубо к описанию термодинамических процессов не привели к значимым результатам, в частности из-за того, что взаимодействия, ответственные за термодинамические процессы, нельзя описать включением в гамильтониан аддитивных добавок [3].

Интерес к обобщению соотношений взаимности Онзагера на открытые нелинейные системы, возникший в последние десятилетие, во многом стимулирован перспективами спинтроники [4], стрейнтроники [5] и спиновой калоритроники [6]. Наблюдение аномального эффекта Эттингаузена [7], переключение знака эффекта Пельтье совместным воздействием магнитного поля и механического напряжения [8,9] создают основу для эффективных систем спин-теплового транспорта. При этом обнаружены существенные нарушения соотношений взаимности между спин-зависимыми эффектами Пельтье и Зеебека, обусловленные нелинейностью вольт-амперной характеристики при больших транспортных токах [10]. Для нелинейных систем соотношения взаимности удается получить только для частных случаев, например, для замкнутых систем в постоянном и однородном магнитном поле [11]. Экспериментально показано, что эти соотношения выполняются в пределах погрешности измерений [12]. Поскольку элементы спинтроники и калоритроники являются открытыми нелинейными системами, функционирующими в неоднородных и нестационарных магнитных, электрических и температурных полях, представляют интерес общие соотношения взаимности для таких систем.

В современной квантовой механике динамику открытых систем описывают уравнением Линдблада для матрицы плотности системы, полученным исключением переменных окружения (резервуара) [13]. При этом оператор, ответственный за обмен энергией с резервуаром, имеет вид супероператора Линдблада. Однако точный вывод такого оператора, гарантирующий как сохранение следа матрицы плотности, так и ее положительную определенность, очень сложен. На практике используют различные приближения и феноменологические соображения, например, полагают, что супероператор для взаимодействующих подсистем является суммой супероператоров для невзаимодействующих подсистем. В работе [14] показано, что такой подход не гарантирует выполнение законов термодинамики, а средние значения операторов могут по порядку величины отличаться от правильных. Альтернативой является анализ открытых систем методом квазиравновесного оператора плотности [15]. Термодинамические процессы при этом рассматриваются как возмущения квазиравновесного оператора, которые входят аддитивно в уравнение Неймана для оператора плотности. Такой подход применим для нелинейной среды при наличии спиновых токов и потоков тепла. 


\section{1. Локально квазиравновесное распределение}

Рассмотрим находящуюся в неоднородных и переменных электрическом и магнитном полях открытую систему, состоящую из нескольких сортов (компонент) взаимодействующих частиц, тождественных в каждой компоненте. Система обменивается энергией и веществом с термостатами и резервуарами частиц через контакты, т. е. участки $S_{k}$ ограничивающей систему поверхности $S$, в которых она контактирует с внешней средой. Пусть гамильтониан системы $\hat{H}$ и ее наблюдаемые $\hat{D}_{i}$, не зависящие в представлении Шредингера явно от времени, можно описать квазилокальными операторами плотности

$$
\hat{H}(t)=\int_{V} \hat{h}(t, \mathbf{r}) d^{3} r+\hat{H}_{r}(t), \quad \hat{D}_{i}(t)=\int_{V} \hat{d}_{i}(t, \mathbf{r}) d^{3} r,
$$

где $\hat{H}_{r}-$ релаксационный гамильтониан взаимодействия системы с внешней средой, $V$ - объем системы. Квазилокальным является оператор $\hat{d}(t, \mathbf{r})$, матричный элемент которого в координатном представлении $d\left(t, \mathbf{r}, \mathbf{r}^{\prime}, \mathbf{r}^{\prime \prime}\right)$ быстро убывает при удалении хотя бы одной из точек $\mathbf{r}^{\prime}$ и $\mathbf{r}^{\prime \prime}$ от точки $\mathbf{r}$ [16]. В (1) и далее используется представление взаимодействия.

Пусть частицы $i$-й компоненты имеют массу $m_{i}$, заряд $q_{i}$, гиромагнитное отношение для спина $\gamma_{i}$ и спин $s_{i}$. Если частицы связаны кулоновским взаимодействием, то можно выбрать в представлении вторичного квантования $[15,16]$

$$
\begin{aligned}
& \hat{h}(t, \mathbf{r})=\frac{1}{2 m_{i}}\left(\hat{\mathbf{p}}_{i}+\frac{q_{i}}{c} \hat{\mathbf{A}}(t, \mathbf{r})\right) \hat{\Psi}_{i \sigma}^{+}(t, \mathbf{r})\left(\hat{\mathbf{p}}_{i}-\frac{q_{i}}{c} \hat{\mathbf{A}}(t, \mathbf{r})\right) \\
& \times \hat{\Psi}_{i \sigma}(t, \mathbf{r})-\hbar \gamma_{i} \hat{\Psi}_{i \sigma}^{+}(t, \mathbf{r}) \mathbf{s}_{i \sigma \sigma^{\prime}} \hat{\Psi}_{i \sigma^{\prime}}(t, \mathbf{r}) \mathbf{B}(r, \mathbf{r}) \\
& \quad+q_{i} \hat{\Psi}_{i \sigma}^{+}(t, \mathbf{r}) \hat{\Psi}_{i \sigma}(t, \mathbf{r}) \hat{\varphi}(t, \mathbf{r})-\hat{d}_{i}(t, \mathbf{r}) f_{i}(t, \mathbf{r}) .
\end{aligned}
$$

Здесь $\hat{\mathbf{A}}(t, \mathbf{r})$ - оператор векторного потенциала магнитного поля,

$$
\hat{\varphi}(t, \mathbf{r})=\int \hat{\Psi}_{j \sigma}^{+}\left(t, \mathbf{r}^{\prime}\right) \frac{q_{j}}{\left|\mathbf{r}-\mathbf{r}^{\prime}\right|} \hat{\Psi}_{j \sigma}\left(t, \mathbf{r}^{\prime}\right) d^{3} r^{\prime}+\varphi(t, \mathbf{r})
$$

- оператор потенциала электрического поля; $\varphi(t, r)-$ потенциал электрического поля, создаваемого внешними по отношению к рассматриваемой системе источниками; $f_{i}(t, \mathbf{r})$ - распределения заданных механических сил, соответствующих наблюдаемым $D_{i} ; \hat{\Psi}_{i \sigma}(\mathbf{r})-$ полевой оператор частиц $i$-й компоненты; $\sigma-$ спиновая переменная; $\mathbf{s}_{i \sigma \sigma^{\prime}}-$ оператор спина. В точке $\mathbf{r}$ находится частица $i$-й компоненты, в точке $\mathbf{r}^{\prime}$ находится частица $j$-й компоненты. Операторное представление потенциалов обусловлено тем, что они создаются не только внешними источниками, но и частицами системы. Поэтому они не являются заданными функциями времени и координат, а зависят от операторов этих частиц.
Соответственно в представлении взаимодействия

$$
\begin{gathered}
\hat{c}_{i}(t, \mathbf{r})=\hat{\Psi}_{i \sigma}^{+}(t, \mathbf{r}) \hat{\Psi}_{i \sigma}(t, \mathbf{r}), \\
\hat{s}_{i \alpha}(t, \mathbf{r})=\hat{\Psi}_{i \sigma}(t, \mathbf{r}) s_{i \alpha \sigma \sigma^{\prime}} \hat{\Psi}_{i \sigma^{\prime}}(t, \mathbf{r}), \\
\hat{\pi}_{i \alpha}(t, \mathbf{r})=-\frac{i \hbar}{2}\left(\frac{\partial \hat{\Psi}_{i \sigma}(t, \mathbf{r})}{\partial r_{\alpha}} \hat{\Psi}_{i \sigma}(t, \mathbf{r})\right. \\
\left.-\hat{\Psi}_{i \sigma}^{+}(t, \mathbf{r}) \frac{\partial \hat{\Psi}_{i \sigma}(t, \mathbf{r})}{\partial r_{\alpha}}\right)
\end{gathered}
$$

- операторы плотностей частиц и $\alpha$-й проекции спина и импульса частиц $i$-й компоненты (в формулах (4) нет суммирования по $i$ ).

Для перехода к континуальному описанию системы усредним плотность гамильтониана (2) по физически малому объему $v \in V$ с центром в точке $\mathbf{r}$, содержащему достаточно частиц для усреднения. Разложим потенциалы в ряд Тейлора вблизи точки $\mathbf{r}$. Считая объем $v$ малым, ограничимся в его пределах вторым членом разложения. В кулоновской калибровке и дипольном приближении получим

$$
\begin{aligned}
\hat{\varphi}\left(t, \mathbf{r}+\mathbf{r}^{\prime}\right) & =\hat{\varphi}(t, \mathbf{r})+\mathbf{r}^{\prime} \nabla \hat{\varphi}(t, \mathbf{r}), \quad \mathbf{r} \in v, \\
\hat{\mathbf{A}}\left(t, \mathbf{r}+\mathbf{r}^{\prime}\right) & =\hat{\mathbf{A}}(t, \mathbf{r})+\left(\mathbf{r}^{\prime} \nabla\right) \hat{\mathbf{A}}(t, \mathbf{r}) \\
& =\hat{\mathbf{A}}(t, \mathbf{r})+\left[\hat{\mathbf{B}}(t, \mathbf{r}) \times \mathbf{r}^{\prime}\right] / 2 .
\end{aligned}
$$

Подставим разложение (5) в правую часть формулы (2). Пренебрежем квадратичными по магнитному полю величинами и добавим полную производную по времени величины $\hat{\mathbf{P}}(\mathbf{r})) \hat{\mathbf{A}}(t, \mathbf{r})$. После стандартных преобразований для суммы первого, второго и третьего слагаемых получим

$$
\begin{aligned}
-\frac{\hbar^{2}}{2 m_{i}} \hat{\Psi}_{i \sigma}^{+}(\mathbf{r}) \Delta \hat{\Psi}_{i \sigma}(\mathbf{r})-\hat{\mathbf{M}}(\mathbf{r}) \hat{\mathbf{B}}(t, \mathbf{r}) \\
-\hat{\mathbf{P}}(\mathbf{r}) \hat{\mathbf{E}}(t, \mathbf{r})-\hat{\rho}_{e}(t, \mathbf{r}) \hat{\varphi}(\mathbf{r})
\end{aligned}
$$

Здесь

$$
\begin{gathered}
\frac{1}{v} \int_{v} q_{i} \hat{\Psi}_{i \sigma}^{+}\left(t, \mathbf{r}+\mathbf{r}^{\prime}\right) \hat{\Psi}_{i \sigma}\left(t, \mathbf{r}+\mathbf{r}^{\prime}\right) d^{3} r^{\prime}=\hat{\rho}_{0}(t, \mathbf{r})+\hat{\rho}_{e}(t, \mathbf{r}), \\
\hat{\mathbf{P}}(t, \mathbf{r})=\frac{1}{v} \int_{v} q_{i} \hat{\Psi}_{i \sigma}^{+}\left(t, \mathbf{r}+\mathbf{r}^{\prime}\right) \mathbf{r}^{\prime} \hat{\Psi}_{i \sigma}\left(t, \mathbf{r}+\mathbf{r}^{\prime}\right) d^{3} r^{\prime}, \\
\hat{\mathbf{M}}(t, \mathbf{r})=\frac{1}{v} \int_{v}\left\{-\frac{i \hbar q_{i}}{2 c m_{i}} \hat{\Psi}_{i \sigma}\left(t, \mathbf{r}+\mathbf{r}^{\prime}\right)\left[\mathbf{r}^{\prime} \times \frac{\partial}{\partial \mathbf{r}}\right] \hat{\Psi}_{i \sigma}\left(t, \mathbf{r}+\mathbf{r}^{\prime}\right)\right. \\
\left.+\hbar \gamma_{i} \hat{\Psi}_{i \sigma}^{+}\left(t, \mathbf{r}+\mathbf{r}^{\prime}\right) \mathbf{s}_{i \sigma \sigma^{\prime}} \hat{\Psi}_{i \sigma^{\prime}}\left(t, \mathbf{r}+\mathbf{r}^{\prime}\right)\right\} d^{3} r^{\prime}
\end{gathered}
$$

- операторы невозмущенной плотности зарядов, возмущения плотности зарядов, поляризации, т.е. плотности дипольного момента, и намагниченности, т.е. плотности магнитного момента соответственно. Если в 
невозмущенном состоянии система является локально электрически нейтральной, то $\hat{\rho}_{0}(t, \mathbf{r})=0$.

Считая, что усреднение по объему $v$, содержащему много частиц, эквивалентно квантовому среднему, заменим средний по объему оператор возмущения плотности заряда (7) его квантовым средним $\rho(t, \mathbf{r})=\left\langle\hat{\rho}_{e}(t, \mathbf{r})\right\rangle$, которое будем считать заданной классической функцией, определяемой взаимодействием системы с термостатами. Тогда формула (2) с учетом соотношений (5) принимает вид

$$
\hat{h}(t, \mathbf{r})=\hat{h}_{0}(t, \mathbf{r})-\hat{d}_{i}(t, \mathbf{r}) f_{i}(t, \mathbf{r}) .
$$

Здесь

$$
\hat{h}_{0}(t, \mathbf{r})=-\frac{\hbar^{2}}{2 m_{i}} \hat{\Psi}_{i \sigma}^{+}(t, \mathbf{r}) \Delta \hat{\Psi}_{i \sigma}(t, \mathbf{r})
$$

- оператор плотности стационарного невозмущенного гамильтониана $\hat{H}_{0}$.

Операторы $\hat{\mathbf{E}}(t, \mathbf{r})$ и $\hat{\mathbf{B}}(t, \mathbf{r})$ в уравнении (6) - это операторы эффективных полей, действующих на частицу, находящуюся в точке r. Они являются суммами полей, создаваемых внешними по отношению к системе источниками, и полей, создаваемых всеми частицами системы, кроме рассматриваемой. Если источниками частиц являются термостаты, то можно считать, что их состояние не меняется при взаимодействии с системой. Тогда создаваемые внешними источниками поля $\mathbf{E}(t, \mathbf{r})$, $\mathbf{H}(t, \mathbf{r})$ и плотность заряда $\rho(t, \mathbf{r})$ можно считать заданными классическими функциями. Если изменением поляризации и намагниченности в пределах малого объема $v$ можно пренебречь, то можно считать, что эффективное поле - это поле в маленькой сферической полости с центром в точке $\mathbf{r}$ :

$$
\begin{gathered}
\hat{\mathbf{E}}(t, \mathbf{r})=\mathbf{E}(t, \mathbf{r})+4 \pi \hat{\mathbf{P}}(t, \mathbf{r}) / 3, \\
\hat{\mathbf{B}}(t, \mathbf{r})=\mathbf{H}(t, \mathbf{r})+4 \pi \hat{\mathbf{M}}(t, \mathbf{r}) / 3 .
\end{gathered}
$$

В этом случае можно включить слагаемое $-4 \pi \hat{\mathbf{P}}^{2}(t, \mathbf{r}) / 3-4 \pi \hat{\mathbf{M}}^{2}(t, \mathbf{r}) / 3$ в плотность невозмущенного гамильтониана (9). Из второй и третьей формул (7) видно, что при этом он включает в себя дипольное кулоновское взаимодействие зарядов, а также магнитодипольное, спин-орбитальное и спинспиновое (обменное) взаимодействие всех заряженных частиц системы. Кулоновское взаимодействие зарядов между собой в соответствии с формулой (2) учтено слагаемым $\hat{\varphi}(t, \mathbf{r}) \rho(t, \mathbf{r})$, которое рассматривается как возмущение. В число наблюдаемых $\hat{d}_{i}(t, \mathbf{r})$ включены потенциал $\hat{\varphi}(t, \mathbf{r})$, проекции поляризации $\hat{P}_{\alpha}(t, \mathbf{r})$ и намагниченности $\hat{M}_{\alpha}(t, \mathbf{r})$. Им соответствуют заданные механические силы $\rho(t, \mathbf{r}), E_{\alpha}(t, \mathbf{r}), H_{\alpha}(t, \mathbf{r})$.

Внешняя среда имеет число степеней свободы много большее, чем система, и ее состояние не меняется при взаимодействии с системой. Тогда можно считать, что гамильтониан релаксации системы с внешней средой зависит не от операторов внешней среды, а от их квантовых средних, т.е. классических величин, которые являются случайными функциями времени. В этом случае и гамильтониан релаксации системы с внешней средой можно считать случайной функцией времени и наблюдаемых только системы. Динамика оператора плотности $\hat{\rho}$ и наблюдаемых такой системы с учетом уравнений (1) и (8) описывается замкнутыми уравнениями Неймана

$$
\begin{gathered}
i \hbar \frac{\partial \hat{\rho}}{\partial t}=-\int_{V} f_{i}(t, \mathbf{r})\left[\hat{d}_{i}(t, \mathbf{r}), \hat{\rho}\right] d^{3} r+\left[\hat{H}_{r}, \hat{\rho}\right], \\
i \hbar \frac{\partial \hat{d}_{i}}{\partial t}=\left[\hat{d}_{i}\left(t, \mathbf{r}, \hat{H}_{0}\right)\right], \quad \hat{H}_{0}=\int_{V} \hat{h}_{0}(t, \mathbf{r}) d^{3} r .
\end{gathered}
$$

В отсутствии внешних механических воздействий в системе устанавливается стационарное локально квазиравновесное распределение с оператором плотности [15]

$$
\begin{gathered}
\hat{\rho}^{q}(t)=\exp \left\{-\Phi(t)-\int_{V} \theta(t, \mathbf{r})\left(\hat{h}_{0}(t, \mathbf{r})\right.\right. \\
\left.\left.-\mu_{i}(t, \mathbf{r}) \hat{c}_{i}(t, \mathbf{r})+\hbar \gamma_{i} \hat{s}_{i \alpha}(t, \mathbf{r}) B_{\alpha}(t, \mathbf{r})\right) d^{3} r\right\}, \\
\Phi(t)=\ln \operatorname{Sp} \exp \left\{-\int_{V} \theta(t, \mathbf{r})\left(\hat{h}_{0}(t, \mathbf{r})\right.\right. \\
\left.\left.-\mu_{i}(t, \mathbf{r}) \hat{c}_{i}(t, \mathbf{r})+\hbar \gamma_{i} \hat{s}_{i \alpha}(t, \mathbf{r}) B_{\alpha}(t, \mathbf{r})\right) d^{3} r\right\} .
\end{gathered}
$$

Здесь $\Phi$ - функционал Масье-Планка для квазиравновесной системы во внешних полях [17], $\theta(t, \mathbf{r})=1 /(k T(t, \mathbf{r})), \quad k-$ постоянная Больцмана, $T(t, \mathbf{r})$ - локальная температура, $\mu_{i}(t, \mathbf{r})$ - локальный химический потенциал частиц $i$-й компоненты. Неоднородное и нестационарное распределение функций и операторов в (11) и (12) может быть создано при контакте системы с несколькими различными термостатами и резервуарами.

\section{2. Термодинамические силы и потоки}

В представлении вторичного квантования введем для каждой компоненты полную ортонормированную систему „одночастичных“ функций $\Psi_{p}^{i}(\mathbf{r}, t)$ с дискретным индексом $p$. В пространстве волновых функций вторичного квантования $\Phi\left(n_{1}^{1}, \ldots, n_{p}^{i}, \ldots\right)$, где $n_{p}^{i}-$ число заполнения $p$-го одночастичного состояния $i$-й компоненты, введем базис собственных функций невозмущенного гамильтониана $\hat{H}_{0} \Phi_{k}\left(\ldots n_{p}^{i} \ldots\right)=E_{k} \Phi_{k}\left(\ldots n_{p}^{i} \ldots\right)$.

Решение второго уравнения Неймана (10) имеет вид

$$
\begin{gathered}
\hat{d}(t, \mathbf{r})=\exp \left(i \hat{H}_{0} t / \hbar\right) \hat{d}(0, \mathbf{r}) \exp \left(-i \hat{H}_{0} t / \hbar\right), \\
d_{k m}(t, \mathbf{r})=\exp \left(i \omega_{k m} t^{\prime}\right) d_{k m}\left(t-t^{\prime} \mathbf{r}\right)
\end{gathered}
$$


Здесь $\omega_{k m}=\left(E_{k}-E_{m}\right) / \hbar$. Соответственно с учетом эрмитовости наблюдаемых

$$
\begin{gathered}
\hat{d}_{i}(-t, \mathbf{r})=\varepsilon_{i} \hat{d}_{i}^{*}(t, \mathbf{r}), \\
d_{i k m}(-t, \mathbf{r})=\varepsilon_{i} d_{i k m}^{*}(t, \mathbf{r})=\varepsilon_{i} d_{i m k}(t, \mathbf{r}) .
\end{gathered}
$$

Здесь $\varepsilon_{i}=1$, если $i$-я наблюдаемая не меняет знак при инверсии времени как поляризация, и ее оператор в представлении Шредингера действительный, $\varepsilon_{i}=-1$, если $i$-я наблюдаемая меняет знак при инверсии времени как намагниченность, и ее оператор в представлении Шредингера мнимый.

Введем векторы $\mathbf{F}(t, \mathbf{r})=\left\{\ldots f_{i}(t, \mathbf{r}), \ldots\right\}=\mathbf{F}(-t, \mathbf{r})$ и $\tilde{\mathbf{F}}\left(t, \mathbf{r}=\left\{\ldots \varepsilon_{i} f_{i}(t, \mathbf{r}), \ldots\right\}\right.$. Будем на этом этапе считать компоненты магнитной индукции, от которых явно зависит квазиравновесное распределение (11), отдельными силами, не всходящими в вектор механических сил $\mathbf{F}$. Тогда квазиравновесное распределение (11) не зависит явно от механических сил. Поскольку $\hat{s}_{i \alpha}^{*}(t, \mathbf{r})=-\hat{s}_{i \alpha}(t, \mathbf{r})$, из первого уравнения (11) получаем

$$
\hat{\rho}^{q *}(-\mathbf{B}(t, \mathbf{r}), \mathbf{T}(t, \mathbf{r}))=\hat{\rho}^{q}(\mathbf{B}(t, \mathbf{r}), \mathbf{T}(t, \mathbf{r})) .
$$

Здесь $\mathbf{T}(t, \mathbf{r})=\left\{T(t, \mathbf{r}), \mu_{1}(t, \mathbf{r}), \ldots\right\}$. Из первого уравнения (10) получаем

$$
\hat{\rho}(-t, \mathbf{F}(t, \mathbf{r}))=\hat{\rho}^{*}(t, \tilde{\mathbf{F}}(t, \mathbf{r}))
$$

Выполним в соотношении

$$
d_{i}(t, \mathbf{r})=\operatorname{Sp}\left\{\hat{\rho}(t, \mathbf{F}(t, \mathbf{r})) \hat{d}_{i}(t, \mathbf{r})\right\}
$$

инверсию времени. С учетом соотношений (13) и (15) получаем $\varepsilon_{i} d_{i}(t, \mathbf{r})=\operatorname{Sp}\left\{\hat{\rho}^{*}(t, \tilde{\mathbf{F}}(t, \mathbf{r})) \varepsilon_{i} \hat{d}_{i}^{*}(t, \mathbf{r})\right\}$. Из действительности наблюдаемой $d_{i}(t, \mathbf{r})$ следует, что $d_{i}(t, \mathbf{r})=\operatorname{Sp}\left\{\hat{\rho}(t, \tilde{\mathbf{F}}(t, \mathbf{r})) \hat{d}_{i}(t, \mathbf{r})\right\}$. Таким образом,

$$
\hat{\rho}(t, \mathbf{F}(t, \mathbf{r}))=\hat{\rho}(t, \tilde{\mathbf{F}}(t, \mathbf{r})) .
$$

Первое уравнение (10) в приближении марковской релаксации, соответствующему предположению Онзагеpa [1], в матричной форме имеет вид

$$
\begin{gathered}
\rho_{k m}\left(t_{0}, \mathbf{F}, \mathbf{T}, \mathbf{B}\right)=\rho_{k m}^{0}(\mathbf{F}, \mathbf{T}, \mathbf{B}), \\
\frac{\partial \rho_{k m}(t, \mathbf{F}, \mathbf{T}, \mathbf{B})}{\partial t}=\frac{\rho_{k m}^{q}(\mathbf{T}, \mathbf{B})-\rho_{k m}(t, \mathbf{F}, \mathbf{T}, \mathbf{B})}{\tau_{k m}} \\
-\frac{i}{\hbar} \int_{V} f_{j}(t, \mathbf{r})\left(\rho_{k l}(t, \mathbf{F}, \mathbf{T}, \mathbf{B}) d_{j l m}(t, \mathbf{r})\right. \\
\left.-d_{j k l}(t, \mathbf{r}) \rho_{l m}(t, \mathbf{F}, \mathbf{T}, \mathbf{B})\right) d^{3} r .
\end{gathered}
$$

Здесь $\tau_{k m}=\tau_{m k}$ - вещественные положительные времена релаксации, и принято, что в момент времени $t_{0}$ система находилась в квазиравновесном состоянии с матрицей плотности $\rho_{k m}^{0}$. Зависимость функций $\mathbf{F}, \mathbf{T}$ и $\mathbf{B}$ от времени и координат здесь и далее подразумевается.
Будем искать решение уравнения (17) в виде $\rho_{k m}(t, \mathbf{F}, \mathbf{T}, \mathbf{B})=\tilde{\rho}_{k m}(t, \mathbf{F}, \mathbf{T}, \mathbf{B}) \exp \left(i \omega_{k m} t\right) . \quad$ Из второго уравнения (13) следует

$$
\begin{aligned}
& \tilde{\rho}_{k m}\left(t_{0}, \mathbf{F}, \mathbf{T}, \mathbf{B}\right)=\rho_{k m}^{0}(t, \mathbf{T}, \mathbf{B}) \exp \left(-i \omega_{k m} t_{0}\right), \\
& \frac{\partial \tilde{\rho}_{k m}(t, \mathbf{F}, \mathbf{T}, \mathbf{B})}{\partial t} \\
& =\frac{\rho_{k m}^{q}(\mathbf{T}, \mathbf{B}) \exp \left(-i \omega_{k m} t_{0}\right)-\tilde{\rho}_{k m}(t, \mathbf{F}, \mathbf{T}, \mathbf{B})}{\tau_{k m}} \\
& -i \omega_{k m} \tilde{\rho}_{k m}(t, \mathbf{F}, \mathbf{T}, \mathbf{B})-\frac{i}{\hbar} \int_{V} f_{j}(t, \mathbf{r}) \\
& \times\left(\tilde{\rho}_{k l}(t, \mathbf{F}, \mathbf{T}, \mathbf{B}) d_{j l m}(0, \mathbf{r})\right. \\
& \left.-d_{j k l}(0, \mathbf{r}) \tilde{\rho}_{l m}(t, \mathbf{F}, \mathbf{T}, \mathbf{B},)\right) d^{3} r .
\end{aligned}
$$

Выполним в уравнении (18) комплексное сопряжение, заменив $\mathbf{F}(t, \mathbf{r})$ на $-\tilde{\mathbf{F}}(t, \mathbf{r}), \mathbf{B}(t, \mathbf{r})$ на $-\mathbf{B}(t, \mathbf{r})$, но оставив неизменным $\mathbf{T}(t, \mathbf{r})$. С учетом уравнения (14) получаем

$$
\begin{aligned}
& \tilde{\rho}_{k m}^{*}\left(t_{0},-\tilde{\mathbf{F}}, \mathbf{T},-\mathbf{B}\right)=\rho_{k m}^{q}(\mathbf{T}, \mathbf{B}) \exp \left(-i \omega_{m k} t_{0}\right), \\
& \frac{\partial \tilde{\rho}_{k m}^{*}(t,-\tilde{\mathbf{F}}, \mathbf{T},-\mathbf{B})}{\partial t} \\
= & \frac{\tilde{\rho}_{k m}^{q}(t, \mathbf{T}, \mathbf{B}) \exp \left(-i \omega_{m k} t_{0}\right)-\tilde{\rho}_{k m}^{*}(t,-\tilde{\mathbf{F}}, \mathbf{T},-\mathbf{B})}{\tau_{k m}} \\
- & i \omega_{m k} \tilde{\rho}_{k m}^{*}(t,-\tilde{\mathbf{F}}, \mathbf{T},-\mathbf{B})-\frac{i}{\hbar} \int_{V} \varepsilon_{j} f_{j}(t, \mathbf{r}) \\
\times & \left(\tilde{\rho}_{k l}^{*}(t,-\tilde{\mathbf{F}}, \mathbf{T},-\mathbf{B}) d_{j l m}(0, \mathbf{r})\right. \\
- & \left.d_{j k l}(0, \mathbf{r}) \tilde{\rho}_{l m}^{*}(t,-\tilde{\mathbf{F}}, \mathbf{T},-\mathbf{B},)\right) d^{3} r .
\end{aligned}
$$

С учетом второго уравнения (13) из единственности решения задач Коши (18) и (19) следует, что $\tilde{\rho}_{k m}(\mathbf{F}(t, \mathbf{r}), \mathbf{T}(t, \mathbf{r}), \mathbf{B}(t, \mathbf{r}))=\tilde{\rho}_{k m}^{*}(-\tilde{\mathbf{F}}(t, \mathbf{r}), \mathbf{t}(t, \mathbf{r})-\mathbf{B}(t, \mathbf{r}))$ при одновременной замене всех частот $\omega_{k m}$ на $\omega_{m k} . \quad$ С учетом условия (16) получаем $\tilde{\rho}_{k m}(\mathbf{F}(t, \mathbf{r}), \mathbf{T}(t, \mathbf{r}), \mathbf{B}(t, \mathbf{r}))=\tilde{\rho}_{k m}^{*}(-\mathbf{F}(t, \mathbf{r}), \mathbf{T}(t, \mathbf{r}),-\mathbf{B}(t, \mathbf{r}))$. Таким образом, магнитное поле $\mathbf{B}$, от которого явно зависит квазиравновесное распределение (11), можно рассматривать как механическую силу и включить его в вектор F. Тогда с учетом эрмитовости оператора плотности получаем

$$
\tilde{\rho}_{k m}(\mathbf{F}(t, \mathbf{r}), \mathbf{T}(t, \mathbf{r}))=\tilde{\rho}_{m k}(-\mathbf{F}(t, \mathbf{r}) \mathbf{T}(t, \mathbf{r}))
$$


Уравнение (17) эквивалентно интегральному уравнению

$$
\begin{aligned}
& \rho_{k m}(t)=\left(\rho_{k m}^{0}+\rho_{k m}^{q}(t)\right) \exp \left(\frac{t_{0}-t}{\tau_{k m}}\right)-\rho_{k m}^{q}(t) \\
& +\int_{t_{0}}^{t} \exp \left(\frac{t_{0}-t}{\tau_{k m}}\right) \frac{d \rho_{k m}^{q}\left(t^{\prime}\right)}{d t^{\prime}} d t^{\prime}+\frac{i}{\hbar} \int_{V} \int_{t_{0}}^{t} \exp \left(\frac{t^{\prime}-t}{\tau_{k m}}\right) \\
& \times f_{j}\left(t^{\prime}, \mathbf{r}^{\prime}\right)\left(d_{j k l}\left(t^{\prime}, \mathbf{r}^{\prime},\right) \rho_{l m}\left(t^{\prime}\right)-\rho_{k l} d_{j l m}\left(t^{\prime}, \mathbf{r}^{\prime}\right)\right) d t^{\prime} d^{3} r^{\prime} .
\end{aligned}
$$

Здесь в интеграле, содержащем $\rho_{k m}^{q}$, выполнено интегрирование по частям. В соответствии с уравнением (11)

$$
\begin{gathered}
\frac{d \hat{\rho}^{q}(t)}{d t}=\frac{1}{2}\left\{\hat{\rho}^{q}(t) \hat{A}(t)+\hat{A}(t) \hat{\rho}^{q}(t)\right\}, \\
\hat{A}(t)=-\int_{V} \theta(t, \mathbf{r})\left(\frac{\partial \hat{h}_{0}(t, \mathbf{r})}{\partial t}-\mu_{i}(t, \mathbf{r}) \frac{\hat{c}_{i}(t, \mathbf{r})}{\partial t}\right. \\
\left.+\hbar \gamma_{i} B_{\alpha}(t, \mathbf{r}) \frac{\partial \hat{s}_{\alpha i}(t, \mathbf{r})}{\partial t}\right) d^{3} r \\
-\int_{V}\left(\frac{\partial \theta(t, \mathbf{r})}{\partial t} \hat{h}_{0}(t \mathbf{r})-\frac{\partial \theta(t, \mathbf{r}) \mu_{i}(t, \mathbf{r})}{\partial t} \hat{c}_{i}(t, \mathbf{r})\right. \\
\left.+\hbar \gamma_{i} \hat{s}_{\alpha i}(t, \mathbf{r}) \frac{\partial \theta(t, \mathbf{r}) B_{\alpha}(t, \mathbf{r})}{\partial t}\right) d^{3} r-\frac{d \Phi}{d t} .
\end{gathered}
$$

Для квазилокальных операторов плотности можно ввести соответствующие операторы плотностей потоков энергии, вещества и спинового тока $i$-й компоненты, удовлетворяющие уравнению непрерывности [16]:

$$
\begin{gathered}
\frac{\partial \hat{h}}{\partial t}=-\frac{\partial \hat{q}_{\alpha}}{\partial r_{\alpha}}, \quad \frac{\partial \hat{c}_{i}}{\partial t}=-\frac{\partial j_{i \alpha}}{\partial r_{\alpha}}, \\
\frac{\partial \hat{s}_{i \alpha}}{\partial t}=-\frac{\partial v_{i \alpha \beta}}{\partial r_{\beta}}, \quad \alpha=1,2,3 .
\end{gathered}
$$

Соотношения (23) определяют операторы плотностей потоков не однозначно. В качестве дополнительного условия можно потребовать, чтобы при преобразовании Галилея $r \rightarrow \mathbf{r}^{\prime}=\mathbf{r}+\mathbf{v} t$ выполнялись правильные трансформационные свойства

$$
\begin{gathered}
\hat{c}_{i}\left(\mathbf{r}^{\prime}\right) \rightarrow \hat{c}_{i}^{\prime}\left(\mathbf{r}^{\prime}\right)=\hat{c}_{i}\left(\mathbf{r}^{\prime}\right), \quad \hat{h}(\mathbf{r}) \rightarrow \hat{h}^{\prime}\left(\mathbf{r}^{\prime}\right)+v^{2} m_{i} \hat{c}_{i}\left(\mathbf{r}^{\prime}\right) / 2, \\
\hat{\pi}_{i \alpha}(\mathbf{r}) \rightarrow \hat{\pi}_{i \alpha}^{\prime}\left(\mathbf{r}^{\prime}\right)=\hat{\pi}_{i \alpha}\left(\mathbf{r}^{\prime}\right)+v_{\alpha} m_{i} \hat{c}_{i}\left(\mathbf{r}^{\prime}\right) \\
\hat{j}_{i \alpha}\left(\mathbf{r}^{\prime}\right) \rightarrow \hat{j}_{i \alpha}^{\prime}\left(\mathbf{r}^{\prime}\right)=\hat{j}_{i \alpha}\left(\mathbf{r}^{\prime}\right)+v_{\alpha} \hat{c}_{i}\left(\mathbf{r}^{\prime}\right) \\
\hat{q}_{i \alpha}(\mathbf{r}) \rightarrow \hat{q}_{i \alpha}^{\prime}\left(\mathbf{r}^{\prime}\right)=\hat{q}_{i \alpha}\left(\mathbf{r}^{\prime}\right)+v_{\alpha} \hat{h}\left(\mathbf{r}^{\prime}\right) \\
+v^{2} m_{i} \hat{j}_{i \alpha}^{\prime}\left(\mathbf{r}^{\prime}\right) / 2+v_{\alpha} v_{\beta} \hat{\pi}_{\beta}\left(\mathbf{r}^{\prime}\right) .
\end{gathered}
$$

Условиям (23) и (24) удовлетворяет оператор плотности (2) вида [16]:

$$
\hat{q}_{\alpha}(t, \mathbf{r})=\frac{i}{2 \hbar} \int_{V} d^{3} r^{\prime} r_{\alpha}^{\prime} \int_{0}^{1}\left[\hat{h}_{0}\left(t, \mathbf{r}-(1-\xi) \mathbf{r}^{\prime}\right), \hat{h}\left(t, \mathbf{r}+\xi \mathbf{r}^{\prime}\right)\right] d \xi .
$$

Аналогичные соотношения справедливы и для операторов плотности вида (4). Здесь коммутатор во внутреннем интеграле вычисляется с учетом перестановочных соотношений

$$
\Psi_{i \sigma^{\prime}}\left(\mathbf{r}^{\prime}\right) \hat{\Psi}_{i \sigma}^{+} \pm \hat{\Psi}_{i \sigma}(\mathbf{r}) \hat{\Psi}_{i \sigma^{\prime}}\left(\mathbf{r}^{\prime}\right)=\delta\left(\mathbf{r}-\mathbf{r}^{\prime}\right) \delta_{\sigma \sigma^{\prime}},
$$

где верхний знак для фермионов, а нижний - для бозонов. Остальные коммутаторы и антикоммутаторы равны нулю. Кроме того, $\hat{\Psi}_{i \sigma^{\prime}}^{+}\left(\mathbf{r}^{\prime}\right) \Delta_{\mathbf{r}} \hat{\Psi}_{i s}(\mathbf{r})=\Delta_{\mathbf{r}}\left(\hat{\Psi}_{i \sigma^{\prime}}^{+}\left(\mathbf{r}^{\prime}\right) \hat{\Psi}_{i \sigma}(\mathbf{r})\right)$. Тогда с учетом (9)

$$
\begin{gathered}
\hat{j}_{i \alpha}=\frac{\hat{\pi}_{i \alpha}}{m_{i}}, \\
\hat{v}_{i \alpha \beta}=\hat{\Psi}_{i \sigma} s_{i \alpha \sigma \sigma^{\prime}}-\frac{\hat{p}_{i \beta}}{2 m_{i}} \hat{\Psi}_{i \sigma^{\prime}}-\frac{\hat{p}_{i \beta}}{2 m_{i}} \hat{\Psi}_{i \sigma}^{+} s_{i \alpha \sigma \sigma^{\prime}} \hat{\Psi}_{i \sigma^{\prime}} .
\end{gathered}
$$

Подставим в первый интеграл в правой части второго уравнения (22) уравнения (23) и выполним интегрирование по частям

$$
\begin{aligned}
& \int_{V} \theta\left(\frac{\partial \hat{h}_{0}}{\partial t}-\mu_{i} \frac{\partial \hat{c}_{i}}{\partial t}+\hbar \gamma B_{\alpha} \frac{\partial \hat{s}_{\sigma i}}{\partial t}\right) d^{3} r \\
& =-\theta_{k} \hat{Q}_{k}+\theta_{k} \mu_{i k} \hat{I}_{i k}-\hbar \gamma_{i} \theta_{k} B_{\alpha k} \hat{\Sigma}_{i \alpha k} \\
& +\int_{V}\left(\hat{q}_{\alpha} \frac{\partial \theta}{\partial r_{\alpha}}-\hat{j}_{i \alpha} \frac{\partial\left(\theta \mu_{i}\right)}{\partial r_{\alpha}}+\hbar \gamma_{i} \hat{v}_{i \alpha \beta} \frac{\partial\left(\theta B_{\alpha}\right)}{\partial r_{\beta}}\right) d^{3} r .
\end{aligned}
$$

Здесь

$$
\begin{gathered}
\hat{Q}_{k}(t)=\int_{s_{k}} n_{k \beta}(\mathbf{r}) \hat{q}_{\beta}(t, \mathbf{r}) d^{2} r, \\
\hat{I}_{i k}(t)=\int_{s_{k}} n_{k \beta}(\mathbf{r}) \hat{j}_{i \beta}(t, \mathbf{r}) d^{2} r, \\
\Sigma_{i \alpha k}(t)=\int_{s_{k}} n_{k \beta}(\mathbf{r}) v_{i \alpha \beta}(t, \mathbf{r}) d^{2} r
\end{gathered}
$$

- операторы потоков энергии, частиц $i$-й компоненты и $\alpha$-проекции спинового тока частиц $i$-й компоненты через $k$-й контакт соответственно, $\theta_{k}(t)=\theta\left(t, \mathbf{r}_{k}\right)$, $\mu_{i k}(t)=\mu_{i}\left(t, \mathbf{r}_{k}\right), \quad B_{\alpha k}(t)=B_{\alpha}\left(t, \mathbf{r}_{k}\right), \quad \mathbf{r}_{k}-$ координата центра $k$-го контакта, $n_{k \beta}-\beta$-проекция внешней нормали к поверхности $k$-го контакта.

Введем, наряду с механическими наблюдаемыми $\hat{d}_{i}(\mathbf{r})$ и соответствующими им механическими силами $f_{i}(t, \mathbf{r})$, термодинамические наблюдаемые - плотности энергии $\hat{h}_{0}(t, \mathbf{r})$, частиц $i$-й компоненты $\hat{c}_{i}(t, \mathbf{r})$ и проекции их спина $\hat{s}_{i \alpha}(t, \mathbf{r})$, проекции плотностей потока энергии $\hat{q}_{\alpha}(t, \mathbf{r})$ и частиц $i$-й компоненты $\hat{j}_{i \alpha}(t, \mathbf{r})$, компоненты 
тензора плотности спинового тока частиц $i$-й компоненты $\hat{v}_{i \alpha \beta}(t, \mathbf{r})$, потоки энергии $-\hat{Q}_{k}(t)$, частиц $i$-й кмпоненты $\hat{I}_{i k}(t), \alpha$-проекции спинового тока частиц $i$ й компоненты $\hat{\Sigma}_{i \alpha k}$ через $k$-й контакт и соответствующие им термодинамические силы $\partial \theta / \partial t,-\partial\left(\theta \mu_{i}\right) \partial t$, $\partial \theta / \partial r_{\alpha},-\partial\left(\theta \mu_{i}\right) / \partial r_{\alpha}, \hbar \gamma_{i} \partial\left(\theta B_{\alpha}\right) / \partial r_{\beta}, \theta_{k}(t), \theta_{k}(t) \mu_{i k}(t)$, $-\hbar \gamma_{i} \theta_{k} B_{\alpha k}$. Сохраним за всеми наблюдаемыми и силами общие нумерацию и обозначения $\hat{d}_{i}(\mathbf{r})$ и $f_{i}(t, \mathbf{r})$, но в вектор $\mathbf{F}(t, \mathbf{r})$ будем включать только механические силы, а термодинамические силы включим в вектор $\mathbf{T}(t, \mathbf{r})$.

\section{3. Соотношения взаимности}

Устремим момент времени $t_{0}$ в формуле $(21) \kappa-\infty$, тогда первое слагаемое в правой части равно нулю. Введем новую переменную $\tau=t-t^{\prime}$. С учетом формул (22) и (26) уравнение (21) принимает вид

$$
\begin{aligned}
& \rho_{k m}(t)=\frac{i}{\hbar} \int_{V} \int_{0}^{\infty} \exp \left(\frac{-\tau}{\tau_{k m}}\right)\left(d_{j k l}\left(t-\tau, \mathbf{r}^{\prime}\right) \rho_{k l}(t-\tau)\right. \\
& \left.-\rho_{k l}(t-\tau) d_{j l m}\left(t^{\prime}, \mathbf{r}^{\prime}\right)\right) f_{j}\left(t-\tau \mathbf{r}^{\prime}\right) d \tau d^{3} r^{\prime} \\
& -\rho_{k m}^{q}(t)-\int_{0}^{\infty} \exp \left(\frac{-\tau}{\tau_{k m}}\right) \rho_{k m}^{q}(t-\tau) \frac{d \Phi(t-\tau)}{d t} d \tau \\
& -\frac{1}{2} \int_{V} \int_{0}^{\infty} \exp \left(\frac{-\tau}{\tau_{k m}}\right)\left(d_{j k l}\left(t-\tau, \mathbf{r}^{\prime}\right) \rho_{k l}^{q}(t-\tau)\right. \\
& \left.+\rho_{k l}^{q}(t-\tau) d_{j l m}\left(t^{\prime}, \mathbf{r}^{\prime}\right)\right) f_{j}\left(t-\tau \mathbf{r}^{\prime}\right) d \tau d^{3} r^{\prime} .
\end{aligned}
$$

В первом интеграле объединены механические величины, а в третьем - термодинамические.

Для среднего значения наблюдаемых из формулы (27) получаем

$$
\begin{aligned}
& d_{i}(t, \mathbf{r}, \mathbf{F}, \mathbf{T})=\rho_{k m}(t, \mathbf{F}, \mathbf{T}) d_{i m k}(t, \mathbf{r}, \mathbf{F}, \mathbf{T})=d_{i}^{q}(t, \mathbf{r}) \\
& +\int_{V} \int_{0}^{\infty} \chi_{i j}\left(\tau, \mathbf{r}, \mathbf{r}^{\prime}, \mathbf{F}, \mathbf{T}\right) f_{j}(t-\tau, \mathbf{r}) d \tau d^{3} r^{\prime} .
\end{aligned}
$$

Здесь первое слагаемое в правой части (28) имеет вид

$$
\begin{aligned}
& d_{i}^{q}(t, \mathbf{r}, \mathbf{F}, \mathbf{T})=-\rho_{k m}^{q}(t) d_{i m k}(\mathbf{r}, t) \\
& -\int_{0}^{\infty} \exp \left(\frac{-\tau}{\tau_{k m}}\right) \rho_{k m}^{q}(t-\tau) d_{i m k}(t, \mathbf{r}) \frac{d \Phi(t-\tau)}{d t} d \tau
\end{aligned}
$$

и описывает квазиравновесное значение наблюдаемой.

Второе слагаемое в (28) описывает отклик нелинейной системы на механические и термодинамические воздействия. Для механического воздействия функция отклика с учетом второго уравнения (13) при $t^{\prime}=t-\tau / 2$ имеет вид формулы Кубо [2]

$$
\begin{aligned}
& \chi\left(\tau, \mathbf{r}, \mathbf{r}^{\prime}, \mathbf{F}(t, \mathbf{r}), \mathbf{T}(t, \mathbf{r})\right)=\frac{i \rho_{k m}(t-\tau, \mathbf{F}(t, \mathbf{r}), \mathbf{T}(t, \mathbf{r}))}{\hbar \exp \left(\tau / \tau_{k m}\right)} \\
& \times\left\{d_{i m l}(t, \mathbf{r}) d_{j l k}\left(t-\tau, \mathbf{r}^{\prime}\right)-d_{j m l}\left(t-\tau, \mathbf{r}^{\prime}\right) d_{i l k}(t, \mathbf{r})\right\} \\
& =\frac{i \tilde{\rho}_{k m}(\mathbf{F}(t, \mathbf{r}), \mathbf{T}(t, \mathbf{r}))}{\hbar \exp \left(\tau / \tau_{k m}\right)} \exp \left(i \omega_{m k} \frac{\tau}{2}\right)\left\{d_{i m l}\left(\frac{\tau}{2}, \mathbf{r}\right)\right. \\
& \left.\times d_{j l k}\left(-\frac{\tau}{2}, \mathbf{r}\right)-d_{j m l}\left(-\frac{\tau}{2}, \mathbf{r}^{\prime}\right) d_{i l k}\left(\frac{\tau}{2}, \mathbf{r}\right)\right\} .
\end{aligned}
$$

Для термодинамического воздействия получаем

$$
\begin{aligned}
& \chi_{i j}\left(\tau, \mathbf{r}, \mathbf{r}^{\prime}, \mathbf{F}(t, \mathbf{r}), \mathbf{T}(t, \mathbf{r})\right)=-\frac{\tilde{\rho}_{k m}^{q}(\mathbf{F}(t, \mathbf{r}), \mathbf{T}(t, \mathbf{r}))}{2 \exp \left(\tau / \tau_{k m}\right)} \\
& \times \exp \left(i \omega_{m k} \tau / 2\right)\left\{d_{i m l}(\tau / 2, \mathbf{r}) d_{j l k}\left(-\tau / 2, \mathbf{r}^{\prime}\right)\right. \\
& \left.+d_{j m l}\left(-\tau / 2, \mathbf{r}^{\prime}\right) d_{i l k}(\tau / 2, \mathbf{r})\right\} .
\end{aligned}
$$

Если термодинамическим откликом является поток через $k$-й контакт, то в формулах $(28)-(30)$ берется $\mathbf{r}=\mathbf{r}_{k}$. Если термодинамическим воздействием является температура и (или) химический потенциал $k$-го контакта, то в формулах (28) и (31) берется $\mathbf{r}^{\prime}=\mathbf{r}_{k}$, при этом интегрирование по $\mathbf{r}^{\prime}$ в формуле (28) отсутствует.

Для механической реакции на механическое воздействие с учетом соотношений (20) из формулы (30) получаем

$$
\begin{aligned}
& \chi_{j i}\left(\tau, \mathbf{r}, \mathbf{r}^{\prime},-\mathbf{F}(t, \mathbf{r}), \mathbf{T}(t, \mathbf{r})\right)=\frac{i \tilde{\rho}_{m k}(\mathbf{F}(t, \mathbf{r}), \mathbf{T}(t, \mathbf{r}))}{\hbar \exp \left(\tau / \tau_{k m}\right)} \\
& \times \exp \left(i \omega_{k m} \tau / 2\right)\left\{d_{j m l}(\tau / 2, \mathbf{r}) d_{i l k}\left(-\tau / 2, \mathbf{r}^{\prime}\right)\right. \\
& \left.-d_{i m l}\left(-\tau / 2 \mathbf{r}^{\prime}\right) d_{j l k}(\tau / 2, \mathbf{r})\right\} .
\end{aligned}
$$

Заменив $k \leftrightarrow m$ с учетом второго уравнения (14) и симметрии матрицы $\tau_{k m}$, получаем

$$
\begin{aligned}
& \chi_{j i}\left(\tau, \mathbf{r}, \mathbf{r}^{\prime},-\mathbf{F}(t, \mathbf{r}), \mathbf{T}(t, \mathbf{r})\right)=\frac{i \tilde{\rho}_{k m}(\mathbf{F}(t, \mathbf{r}), \mathbf{T}(t, \mathbf{r}))}{\hbar \exp \left(\tau / \tau_{k m}\right)} \\
& \times \exp \left(i \omega_{k m} \tau / 2\right) \varepsilon_{i} \varepsilon_{j}\left\{d_{j i k}(-\tau / 2, \mathbf{r}) d_{i m l}\left(\tau / 2, \mathbf{r}^{\prime}\right)\right. \\
& \left.-d_{i l k}\left(\tau / 2 \mathbf{r}^{\prime}\right) d_{j m l}(0, \mathbf{r})\right\} .
\end{aligned}
$$

Для термодинамической реакции на термодинамические воздействия из формулы (31) аналогично получаем

$$
\begin{aligned}
& \chi_{j i}\left(\tau, \mathbf{r}, \mathbf{r}^{\prime},-\mathbf{F}(t, \mathbf{r}), \mathbf{T}(t, \mathbf{r})\right)=-\frac{\tilde{\rho}_{k m}^{q}(\mathbf{F}(t, \mathbf{r}), \mathbf{T}(t, \mathbf{r}))}{2 \exp \left(\tau / \tau_{k m}\right)} \\
& \times \exp \left(i \omega_{m k} \tau / 2\right) \varepsilon_{i} \varepsilon_{j}\left\{d_{i m l}(\tau / 2, \mathbf{r}) d_{j i k}\left(-\tau / 2, \mathbf{r}^{\prime}\right)\right. \\
& \left.+d_{j m l}\left(-\tau / 2 \mathbf{r}^{\prime}\right) d_{i l k}(\tau / 2, \mathbf{r})\right\} .
\end{aligned}
$$

Из сравнения формул (30) с (32) и (31) с (33) получаем соотношения взаимности для функции механического отклика открытой нелинейной системы на механические 
воздействия и термодинамического отклика на термодинамические воздействия

$$
\begin{aligned}
& \chi_{i j}\left(\tau, \mathbf{r}, \mathbf{r}^{\prime} \mathbf{F}(t, \mathbf{r}) \mathbf{T}(t, \mathbf{r})\right) \\
& =\varepsilon_{i} \varepsilon_{j} \chi_{j i}\left(\tau, \mathbf{r}^{\prime}, \mathbf{r},-\mathbf{F}(t, \mathbf{r}), \mathbf{T}(t, \mathbf{r})\right)
\end{aligned}
$$

В соотношениях (30)-(34) и далее аргумент функций $\mathbf{F}(t, \mathbf{r})$ и $\mathbf{T}(t, \mathbf{r})$ подразумевает, что оклик зависит от значений сил во все моменты, предшествующие $t$, во всей области $V$. Анализ соотношений взаимности между механическими и термодинамическими величинами, как и квазиравновесного значения (29), видимо, требует определенной информации о структуре системы.

Если механическим откликом являются компоненты поляризации $P_{\alpha}(t, \mathbf{r})$, а механическим воздействием компоненты электрического поля $E_{\alpha}(t, \mathbf{r})$, то функция отклика $\chi_{\alpha \beta}$ является тензором диэлектрической восприимчивости. Если механическим откликом являются компоненты намагниченности $M_{\alpha}(t, \mathbf{r})$, а механическим воздействием - компоненты магнитного поля $H_{\alpha}(t, \mathbf{r})$, то функция отклика $\chi_{\alpha \beta}$ является тензором магнитной восприимчивости. В обоих случаях из формулы (34) следует

$$
\begin{aligned}
& \chi_{\alpha \beta}\left(\tau, \mathbf{r}, \mathbf{r}^{\prime}, \mathbf{E}(t, \mathbf{r}), \mathbf{H}(t, \mathbf{r}), \mathbf{T}(t, \mathbf{r})\right) \\
& =\chi_{\beta \alpha}\left(\tau, \mathbf{r}^{\prime}, \mathbf{r},-\mathbf{E}(t, \mathbf{r}),-\mathbf{H}(t, \mathbf{r}), \mathbf{T}(t, \mathbf{r})\right) .
\end{aligned}
$$

Для функции отклика поляризации на магнитное поле или намагниченности на электрическое поле соответственно

$$
\begin{aligned}
& \chi_{\alpha \beta}\left(\tau, \mathbf{r}, \mathbf{r}^{\prime}, \mathbf{E}(t, \mathbf{r}), \mathbf{H}(t, \mathbf{r}), \mathbf{T}(t, \mathbf{r})\right) \\
& =-\chi_{\beta \alpha}\left(\tau, \mathbf{r}^{\prime}, \mathbf{r},-\mathbf{E}(t, \mathbf{r})-\mathbf{H}(t, \mathbf{r}), \mathbf{T}(t, \mathbf{r})\right) .
\end{aligned}
$$

\section{4. Нелинейная система во внешних полях}

Рассмотрим систему, обменивающуюся энергией и заряженными бесспиновыми частицами одного сорта через два контакта 1 и 2 с температурами $T_{1}$ и $T_{2}$ соответственно. Для заряженных частиц химический потенциал и поток пропорционален электрическому потенциалу и току соответственно. Если источниками частиц являются термостаты, можно считать, что их состояние не меняется при взаимодействии с системой. Тогда токи через контакты можно считать заданными функциями времени и считать их термодинамическими силами. Понимая в дальнейшем под током и потенциалом, который принимается равным нулю на бесконечности, именно электрические величины, выберем нумерацию термодинамических величин на контактах: $e \varphi_{1}, e \varphi_{2}, Q_{1}, Q_{2}$, где $e-$ заряд частицы. Им соответствуют термодинамические силы $\theta_{1} I_{1} / e, \theta_{2} I_{2} / e, \theta_{1}$, $\theta_{2}$. Запишем уравнения (28) в стационарном режиме без квазиравновесных составляющих для контактов 1 и 2. Учитывая, что в стационарном режиме $I_{1}=-I_{2}=I$, получаем

$$
\begin{gathered}
e \varphi_{1}=\kappa_{11} I /\left(T_{1} e\right)-\kappa_{12} I /\left(T_{2} e\right)+\kappa_{13} / T_{1}+\kappa_{14} / T_{2}, \\
e \varphi_{2}=\kappa_{21} I /\left(T_{1} e\right)-\kappa_{22} I /\left(T_{2} e\right)+\kappa_{23} / T_{1}+\kappa_{24} / T_{2}, \\
Q_{1}=\kappa_{31} I /\left(T_{1} e\right)-\kappa_{32} I /\left(T_{2} e\right)+\kappa_{33} / T_{1}+\kappa_{34} / T_{2}, \\
Q_{2}=\kappa_{41} I /\left(T_{1} e\right)-\kappa_{42} I /\left(T_{2} e\right)+\kappa_{43} / T_{1}+\kappa_{44} / T_{2} . \\
\kappa_{i j}(\mathbf{F}, \mathbf{T})=\frac{1}{k} \int_{0}^{\infty} \chi_{i j}\left(\tau, \mathbf{r}_{j}^{\prime}, \mathbf{r}_{j}^{\prime \prime}, \mathbf{F}, \mathbf{T}\right) d \tau, \\
\mathbf{r}_{1}^{\prime}=\mathbf{r}_{3}^{\prime}=\mathbf{r}_{1}^{\prime \prime}=\mathbf{r}_{3}^{\prime \prime}=\mathbf{r}_{1}, \\
\mathbf{r}_{2}^{\prime}=\mathbf{r}_{4}^{\prime}=\mathbf{r}_{2}^{\prime \prime}=\mathbf{r}_{4}^{\prime \prime}=\mathbf{r}_{2} .
\end{gathered}
$$

В формулах (23), (35) и далее полагается, что термодинамические потоки при инверсии времени не меняют знак, как и термодинамические потенциалы. Это обеспечивает необратимость термодинамических процессов. Из соотношений (34) и (36) следует, что

$$
\kappa_{i j}(\mathbf{F}, \mathbf{T})=\kappa_{j i}(-\mathbf{F}, \mathbf{T}), \quad i, j=1,2,3,4 .
$$

Источники частиц являются термостатами, достаточно большими, так что при отсутствии возмущений их потенциалы равны потенциалу на бесконечности, т.е. нулю. Если при $I=0$ и $\theta_{1}=\theta_{2}=\theta$ магнитное поле и механические силы не создают тепловых потоков и электрических потенциалов, то $\varphi_{1}==\varphi_{2}=0, Q_{1}=Q_{2}=0$, т. e.

$$
\begin{gathered}
\kappa_{13}(\mathbf{F}, T, 0)=-\kappa_{14}(\mathbf{F}, T, 0), \\
\kappa_{23}(\mathbf{F}, T, 0)=-\kappa_{24}(\mathbf{F}, T, 0), \\
\kappa_{33}(\mathbf{F}, \mathbf{B}, T, 0)=-\kappa_{34}(\mathbf{F}, T, 0), \\
\kappa_{44}(\mathbf{F}, T, 0)=-\kappa_{43}(\mathbf{F}, T, 0) .
\end{gathered}
$$

Полагая $T_{1}=T-\Delta T / 2, T_{2}=T+\Delta T / 2, U=\varphi_{2}-\varphi_{1}$, получим

$$
\begin{aligned}
U & =k\left\{\kappa_{12}(\mathbf{F}, T, I) / T_{1}+\kappa_{21}(\mathbf{F}, \mathbf{T}, \mathbf{I}) / T_{2}-\kappa_{11}(\mathbf{F}, T, I) / T_{1}\right. \\
& \left.-\kappa_{22}(\mathbf{F}, T, I) / T_{2}\right\} I / e+\left\{\kappa_{24}(\mathbf{F}, T, I)+\kappa_{23}(\mathbf{F}, T, I)\right. \\
& \left.-\kappa_{13}(\mathbf{F}, T, I)-\kappa_{14}(\mathbf{F}, T, I)\right\} /(e T) \\
& -\left\{\kappa_{14}(\mathbf{F}, T, I)+\kappa_{23}(\mathbf{F}, T, I)\right\} / \Delta T /\left(e T^{2}\right), \\
Q_{1} & =\frac{\kappa_{31}(\mathbf{F}, T, 0)-\kappa_{32}(\mathbf{F}, T, 0)}{e T} I \\
& -\frac{\partial}{\partial T} \frac{\kappa_{31}(\mathbf{F}, T, I)-\kappa_{32}(\mathbf{F}, T, I)}{2 e T} I \Delta T \\
+ & \frac{\kappa_{31}(\mathbf{F}, T, I)-\kappa_{31}(\mathbf{F}, T, 0)-\kappa_{32}(\mathbf{F}, T, I)+\kappa_{32}(\mathbf{F}, T, 0)}{e I T} I^{2} \\
- & \frac{\kappa_{34}(\mathbf{F}, T, I)}{T^{2}} \Delta T .
\end{aligned}
$$




$$
\begin{aligned}
& Q_{2}=\frac{\kappa_{41}(\mathbf{F}, T, 0)-\kappa_{42}(\mathbf{F}, T, 0)}{e T} I \\
& +\frac{\partial}{\partial T} \frac{\kappa_{41}(\mathbf{F}, T, I)-\kappa_{42}(\mathbf{F}, T, I)}{2 e T} I \Delta T \\
& +\frac{\kappa_{41}(\mathbf{F}, T, I)-\kappa_{41}(\mathbf{F}, T, 0)-\kappa_{42}(\mathbf{F}, T, I)+\kappa_{42}(\mathbf{F}, T, 0)}{e I T} \\
& \times I^{2}+\frac{\kappa_{43}(\mathbf{F}, T, I)}{T^{2}} \Delta T .
\end{aligned}
$$

В уравнении (39) в силу (38) при $I=0$ первые два слагаемые равны нулю, следовательно, третье описывает эффект Зеебека с коэффициентом

$$
Z(\mathbf{F}, T, I)=\left\{\kappa_{14}(\mathbf{F}, T, I)+\kappa_{23}(\mathbf{F}, T, I)\right\} /\left(e T^{2}\right) .
$$

Первые слагаемые в (40) и (41), в силу (37) и (38) равные по величине и противоположные по знаку, описывают линейный по току эффект Пельтье с коэффициентом

$$
\Pi(\mathbf{F}, T)=\left\{\kappa_{41}(\mathbf{F}, T, 0)-\kappa_{42}(\mathbf{F}, T, 0)\right\} /(e T) .
$$

Последние слагаемые, равные в силу (37), описывают теплопроводность. Сложив уравнения (40) и (41) и добавив работу, совершаемую источником против термоэдс, получим с учетом (37) и (38) для мощности, выделяющейся в системе:

$$
W(\mathbf{F}, T, I)=K(\mathbf{F}, T) I \Delta T+R(\mathbf{F}, T, I) I^{2}+D(\mathbf{F}, T, I) \Delta T .
$$

Первое слагаемое в (44) описывает эффект Томпсона с коэффициентом

$$
\begin{aligned}
& K(\mathbf{F}, T)=\frac{\partial}{\partial T} \\
& \times \frac{\kappa_{42}(\mathbf{F}, T, 0)-\kappa_{41}(\mathbf{F}, T, 0)-\kappa_{32}(\mathbf{F}, T, 0)-\kappa_{31}(\mathbf{F}, T, 0)}{2 e T} \\
& -\left\{\kappa_{14}(\mathbf{F}, T, 0)+\kappa_{23}(\mathbf{F}, T, 0)\right\} /\left(e T^{2}\right) .
\end{aligned}
$$

а второе - выделение тепла Джоуля-Ленца на нелинейном сопротивлении системы

$$
\begin{aligned}
& R(\mathbf{F}, T, I) \\
& =\frac{\kappa_{42}(\mathbf{F}, T, I)-\kappa_{42}(\mathbf{F}, T, 0)+\kappa_{32}(\mathbf{F}, T, I)-\kappa_{32}(\mathbf{F}, T, 0)}{e I} \\
& -\frac{\kappa_{41}(\mathbf{F}, T, I)-\kappa_{41}(\mathbf{F}, T, 0)+\kappa_{31}(\mathbf{F}, T, I)-\kappa_{31}(\mathbf{F}, T, 0)}{e I} .
\end{aligned}
$$

Из соотношений (37) и (38) следуют соотношения Томпсона для нелинейной системы

$$
\begin{gathered}
Z(\mathbf{F}, T, 0)=\Pi(-\mathbf{F}, T) / T, \\
K(\mathbf{F}, T)=d \Pi(\mathbf{F}, T) d T+\Pi(-\mathbf{F}, T) / T .
\end{gathered}
$$

При $F=0$, т.е. для линейной системы, получаем классические соотношения [18]

$$
Z(T)=\Pi(T) / T, \quad K(T)=(1 / T) d(T \Pi(T)) / d T .
$$

Третье слагаемое в (44) описывает эффекты Риги-Ледюка и тензокалорический, т. е. влияние магнитного поля и механических напряжений на теплопроводность

$$
\begin{aligned}
& D(\mathbf{F}, T, I)=k \frac{\kappa_{43}(\mathbf{F}, T, I)-\kappa_{43}(-\mathbf{F},-T, I)}{T} \\
& -\frac{\kappa_{14}(\mathbf{F}, T, I)-\kappa_{14}(\mathbf{F}, T, 0)+\kappa_{23}(\mathbf{F}, T, I)-\kappa_{23}(\mathbf{F}, T, 0)}{e T^{2}} \\
& +\frac{\partial}{\partial T} \frac{\kappa_{42}(\mathbf{F}, T, I)-\kappa_{42}(\mathbf{F}, T, 0)-\kappa_{41}(\mathbf{F}, T, I)+\kappa_{41}(\mathbf{F}, T, I)}{2 e T} \\
& -\frac{\partial}{\partial T} \frac{\kappa_{32}(\mathbf{F}, T, I)-\kappa_{32}(\mathbf{F}, T, 0)-\kappa_{31}(\mathbf{F}, T, I)+\kappa_{31}(\mathbf{F}, T, 0)}{2 e T} .
\end{aligned}
$$

Зависимость коэффициента $D$ от магнитного поля описывает эффекты Холла и Гаусса, а от механического напряжения - тензорезистивный эффект. Зависимости коэффициентов Зеебека (42) и Пельтье (43) от магнитного поля описывают соответственно эффекты Нернста и Эттинсгаузена. Зависимости этих величин от механического напряжения описывают соответствующие тензоэффекты.

Если заряженные частицы, которыми система обменивается с термостатами через контакты, имеют упорядоченный спин, то потенциалы и потоки тепла будут создаваться как зарядовыми, так и спиновыми токами. В этом случае к термодинамическим силам на каждом контакте добавятся 3 компоненты спинового тока $\Sigma_{\alpha}$, а к откликам - 3 компоненты магнитного поля $B_{\alpha}$. Условия взаимности (37) по-прежнему выполняются, но индексы $i$ и $j$ пробегают значения от 1 до 10. Соответственно уравнений вида (35) будет 10. Кроме того, даже в стационарном режиме спиновые токи через контакты не равны по величине. Уравнение для средних компонент плотности спинового момента $s_{\alpha}(t, \mathbf{r})=\left\langle\hat{s}_{\alpha(t, \mathbf{r})}\right\rangle \mathrm{c}$ учетом уравнений $(2),(17)$ и (23) имеет вид

$$
\begin{aligned}
& \frac{\partial S_{\alpha}(t, \mathbf{r})}{\partial t}=\operatorname{Sp}\left(\hat{s_{\alpha}}(t, \mathbf{r}) \frac{d \hat{\rho}}{d t}+\frac{\partial \hat{s}_{\alpha}(t, \mathbf{r})}{\partial t} \hat{\rho}\right) \\
& =\frac{s_{\alpha}^{e}(t, \mathbf{r})-s_{\alpha(t, \mathbf{r})}}{T_{\alpha}}-\hbar \gamma[\mathbf{B}(t, \mathbf{r}) \times \mathbf{s}(t, \mathbf{r})]_{\alpha}-\frac{\partial v_{\alpha \beta}(t, \mathbf{r})}{\partial r_{\beta}}
\end{aligned}
$$

Здесь $v_{\alpha \beta}(t, \mathbf{r})=\left\langle\hat{v}_{\alpha \beta}(t, \mathbf{r})\right\rangle-$ тензор плотности спинового тока, для каждой компоненты плотности спина времена релаксации $\tau_{m n}$ заменены усредненным значением $T_{\alpha}$.

Проинтегрируем уравнение (46) по объему среды, считая магнитное поле однородным

$$
\frac{d S_{\alpha}}{d t}+\frac{S_{\alpha}-S_{\alpha}^{e}}{T_{\alpha}}+\hbar \gamma[\mathbf{B} \times \mathbf{S}]_{\alpha}+\Sigma_{\alpha 2}-\Sigma_{\alpha 1} .
$$

Уравнение (47) следует использовать вместе с уравнениями вида (35). Поэтому при наличии спиновых токов соотношения (38) и вытекающие из них соотношения (45) будут иметь более сложную структуру. 
Градиенты температуры и химического потенциала являются термодинамическими силами. Если химический потенциал частиц і-й компоненты зависит от плотностей частиц всех компонент, то из соотношения (28) получаем

$$
\begin{aligned}
& j_{i \alpha}(t, \mathbf{r}, \mathbf{F}, \mathbf{B})=\iint_{V} \int_{0}^{\infty} \frac{\chi_{i \alpha j \beta}\left(\tau, \mathbf{r}, \mathbf{r}^{\prime}, \mathbf{B}, \mathbf{T}\right)}{k T} \frac{d \mu_{j}\left(t-\tau, \mathbf{r}^{\prime}\right)}{d c_{k}} \\
& \times \frac{\partial c_{k}\left(t-\tau, \mathbf{r}^{\prime}\right)}{\partial r_{\beta}} d \tau d^{3} r^{\prime}+\iint_{V}^{\infty} \xi_{i \alpha j \beta}\left(\tau, \mathbf{r}, \mathbf{r}^{\prime}, \mathbf{F}, \mathbf{B}, \mathbf{T}\right) \\
& \times \mu_{j}\left(t-\tau, \mathbf{r}^{\prime}\right) \frac{\partial T\left(t-\tau, \mathbf{r}^{\prime}\right)}{\partial r_{\beta}} d \tau d^{3} r^{\prime} .
\end{aligned}
$$

Первое слагаемое в соотношении (48) является обобщением закона диффузии Фика, второе - закона термодиффузии Людвига-Соре [18]. В соответствии с (37)

$$
\begin{aligned}
& \chi_{i \alpha j \beta}\left(\tau, \mathbf{r}, \mathbf{r}^{\prime}, \mathbf{F}, \mathbf{B}, \mathbf{T}\right)=\chi_{j \beta i \alpha}\left(\tau, \mathbf{r}^{\prime} \mathbf{r},-\mathbf{F},-\mathbf{B}, \mathbf{T}\right), \\
& \xi_{i \alpha j \beta}\left(\tau, \mathbf{r}, \mathbf{r}^{\prime}, \mathbf{F}, \mathbf{B}, \mathbf{T}\right)=\xi_{j \beta i \alpha}\left(\tau, \mathbf{r}^{\prime} \mathbf{r},-\mathbf{F},-\mathbf{B}, \mathbf{T}\right) .
\end{aligned}
$$

Соотношения взаимности для механического отклика на механическое воздействие рассмотрим на примере электрических величин в отсутствие полей, созданных внешними источниками. Тогда единственной механической наблюдаемой в среде является распределение потенциала $\varphi(t, \mathbf{r})$, а соответствующей ему механической силой - плотность свободных зарядов $\rho(t, \mathbf{r})$, которыми система обменивается с термостатами. Предполагая, что термодинамические воздействия отсутствуют, из уравнения (28) для индуцированной электрическим воздействием реакции получаем

$$
\varphi(t, \mathbf{r})=\int_{V} \int_{0}^{\infty} \chi_{11}\left(\tau, \mathbf{r}, \mathbf{r}^{\prime}, \rho, \mathbf{B}, \mathbf{T}\right) \rho\left(t-\tau, \mathbf{r}^{\prime}\right) d \tau d^{3} r^{\prime}
$$

Опуская в дальнейшем индексы у $\chi$, из соотношения (34) получим

$$
\begin{aligned}
& \chi\left(\tau, \mathbf{r}, \mathbf{r}^{\prime}, \rho(t, \mathbf{r}), \mathbf{B}(t, \mathbf{r}), \mathbf{T}(t, \mathbf{r})\right) \\
& =\chi\left(\tau, \mathbf{r}^{\prime}, \mathbf{r},-\rho(t, \mathbf{r}),-\mathbf{B}(t, \mathbf{r}), \mathbf{T}(t, \mathbf{r})\right)
\end{aligned}
$$

Введем время установления системы $\tau_{r}$, такое, что все $\chi_{i j}\left(\tau>\tau_{r}\right) \equiv 0$. С учетом экспоненциального множителя в формуле (30) можно принять, что $\tau_{r}>\max \left(\tau_{n m}\right)$. Разложим в подынтегральном выражении формулы (49) плотность заряда в ряд Тейлора в окрестности точки $t$. В квазистационарном режиме, когда $\tau_{r}\left|d^{3} \rho / d t^{3}\right| \ll\left|d^{2} \rho / d t^{2}\right|$, при $\tau \leq \tau_{r}$ ограничимся третьим слагаемым ряда и продифференцируем по $r_{\alpha}$ с учетом уравнения непрерывности (23):

$$
\begin{aligned}
& \frac{\partial \varphi(t, \mathbf{r})}{\partial r_{\alpha}}=\int_{V} \int_{0}^{\infty} \frac{\partial \chi\left(\tau, \mathbf{r}, \mathbf{r}^{\prime}, \rho, \mathbf{B}, \mathbf{T}\right)}{\partial r_{\alpha}} \rho\left(t, \mathbf{r}^{\prime}\right) d \tau d^{3} r^{\prime} \\
& +\int_{V} \int_{0}^{\infty} \tau \frac{\partial \chi\left(\tau, \mathbf{r}, \mathbf{r}^{\prime}, \rho, \mathbf{B}, \mathbf{T}\right)}{\partial r_{\alpha}} \frac{\partial j_{\beta}\left(t \mathbf{r}^{\prime}\right)}{\partial r_{\beta}^{\prime}} d \tau d^{3} r^{\prime} \\
& -\frac{\partial}{\partial t} \int_{V} \int_{0}^{\infty} \frac{\tau^{2}}{2} \frac{\partial \chi\left(\tau, \mathbf{r}, \mathbf{r}^{\prime}, \rho, \mathbf{B}, \mathbf{T}\right)}{\partial r_{\alpha}} \frac{\partial j_{\beta}\left(t, \mathbf{r}^{\prime}\right)}{\partial r_{\beta}^{\prime}} d \tau d^{3} r^{\prime} .
\end{aligned}
$$

Проинтегрировав второе и третье слагаемые этого уравнения по частям, учитывая, что во внутренней области для квазилокальной функции $\chi(\tau, \mathbf{r} \in V), \mathbf{r}^{\prime} \in S, \rho, \mathbf{B}, \mathbf{T}=0$, получим

$$
\begin{aligned}
& \frac{\partial \varphi(t, \mathbf{r})}{\partial r_{\alpha}}=\int_{V} \int_{0}^{\infty} \frac{\partial \chi\left(\tau, \mathbf{r}, \mathbf{r}^{\prime}, \rho, \mathbf{B}, \mathbf{T}\right)}{\partial r_{\alpha}} \rho\left(t, \mathbf{r}^{\prime}\right) d \tau d^{3} r^{\prime} \\
& -\int_{V} \int_{0}^{\infty} \tau \frac{\partial^{2} \chi\left(\tau, \mathbf{r}, \mathbf{r}^{\prime}, \rho, \mathbf{B}, \mathbf{T}\right)}{\partial r_{\alpha} \partial r_{\beta}^{\prime}} j_{\beta}\left(\tau \mathbf{r}^{\prime}\right) d \tau d^{3} r^{\prime} \\
& +\frac{\partial}{\partial t} \int_{V} \int_{0}^{\infty} \frac{\tau^{2}}{2} \frac{\partial^{2} \chi\left(\tau, \mathbf{r}, \mathbf{r}^{\prime}, \rho, \mathbf{B}, \mathbf{T}\right)}{\partial r_{\alpha} \partial r_{\beta}^{\prime}} j_{\beta}\left(\tau \mathbf{r}^{\prime}\right) d \tau d^{3} r^{\prime}
\end{aligned}
$$

С учетом уравнения Максвелла получим

$$
\begin{gathered}
E_{\alpha}(t, \mathbf{r})=\int_{V} \xi_{\alpha}\left(\mathbf{r}, \mathbf{r}^{\prime}, \rho, \mathbf{B}, \mathbf{T}\right) \rho\left(t, \mathbf{r}^{\prime}\right) d^{3} r^{\prime} \\
+\int_{V} \lambda_{\alpha \beta}\left(\mathbf{r}, \mathbf{r}^{\prime}, \mathbf{j}, \mathbf{B}, \mathbf{T}\right) j_{\beta}\left(t, \mathbf{r}^{\prime}\right) d^{3} r^{\prime}, \\
A_{\alpha}(t, \mathbf{r})=\int_{V} \xi_{\alpha \beta}\left(\mathbf{r}, \mathbf{r}^{\prime}, \mathbf{j}, \mathbf{A}, \mathbf{T}\right) j_{\beta}\left(t, \mathbf{r}^{\prime}\right) d^{3} r^{\prime}, \\
\xi\left(\mathbf{r}, \mathbf{r}^{\prime}, \rho, \mathbf{B}, \mathbf{T}\right)=-\int_{0}^{\infty} \frac{\partial \chi\left(\tau, \mathbf{r}, \mathbf{r}^{\prime}, \rho, \mathbf{B}, \mathbf{T}\right)}{\partial r_{\alpha}} d \tau \\
\lambda_{\alpha \beta}\left(\mathbf{r}, \mathbf{r}^{\prime}, \mathbf{j}, \mathbf{B}, \mathbf{T}\right)=\int_{0}^{\infty} \tau \frac{\partial^{2} \chi\left(\tau, \mathbf{r}, \mathbf{r}^{\prime}, \mathbf{j}, \mathbf{B}, \mathbf{T}\right)}{\partial r_{\alpha} \partial r_{\beta}^{\prime}} d \tau \\
\xi_{\alpha, \beta}\left(\mathbf{r}, \mathbf{r}^{\prime}, \mathbf{j}, \mathbf{A}, \mathbf{T}\right)=-c \int_{0}^{\infty} \frac{\tau^{2}}{2} \frac{\partial^{2} \chi\left(\tau, \mathbf{r}, \mathbf{r}^{\prime}, \mathbf{j}, \mathbf{A}, \mathbf{T}\right)}{\partial r_{\alpha} \partial r_{\beta}^{\prime}} d \tau .
\end{gathered}
$$

В соотношениях (52) и (53) учтено, что задание векторного потенциала А однозначно определяет магнитное поле В, а в силу уравнения (23) распределение плотности тока определяет плотность заряда. Векторы А и $\mathbf{j}$ меняют знак при инверсии координат. Вторая 
производная функции $\chi$ при инверсии координат знак не меняет. Тогда, считая функцию $\chi$ дважды непрерывно дифференцируемой, получаем с учетом соотношений (50) и (52)

$$
\begin{aligned}
& \lambda_{\alpha, \beta}(\mathbf{r}, \mathbf{r} /, \mathbf{j}, \mathbf{b}, \mathbf{T})=\lambda_{\beta \alpha}\left(\mathbf{r}^{\prime}, \mathbf{r},-\mathbf{j},-\mathbf{B}, \mathbf{T}\right), \\
& \xi_{\alpha \beta}\left(\mathbf{r}, \mathbf{r}^{\prime}, \mathbf{j}, \mathbf{A}, \mathbf{T}\right)=\xi_{\beta, \alpha}\left(\mathbf{r}^{\prime}, \mathbf{r},-\mathbf{j},-\mathbf{A}, \mathbf{T}\right) \\
& =\zeta_{\beta \alpha}\left(\mathbf{r}^{\prime}, \mathbf{r}, \mathbf{j}, \mathbf{A}, \mathbf{T}\right) .
\end{aligned}
$$

Первое слагаемое в уравнении (51) представляет собой кулоновское поле, создаваемое распределенным зарядом в неоднородной анизотропной нелинейной среде с пространственной дисперсией. Второе слагаемое в уравнении (51) является обобщением дифференциального закона Ома, а уравнение (52) - аналогом закона Био-Савара.

Рассмотрим динамику энергии системы при воздействии механических сил. Из уравнений (1), (9) и (10) и правила перестановки операторов под знаком шпура получаем

$$
\begin{aligned}
& \frac{d W}{d t}=\frac{d\langle\hat{H}\rangle}{d t}=\mathbf{S p}\left(\frac{\partial \hat{\rho}}{\partial t} \hat{H}\right)-\int_{V} f_{i}(t, \mathbf{r}) \\
& \times \mathbf{S p}\left(\hat{\rho} \frac{\partial \hat{d}_{i}(t, \mathbf{r})}{\partial t}\right) d^{3} r+\mathbf{S p}\left(\hat{\rho} \frac{\partial \hat{H}_{r}}{\partial t}\right) \\
& -\int_{V} \frac{\partial f_{i}(t, \mathbf{r})}{\partial t} \mathbf{S p}\left(\hat{\rho} \hat{d}_{i}(t, \mathbf{r})\right) d^{3} r \\
& =-\int_{V} \frac{\partial f_{i}(t, \mathbf{r})}{\partial t} d_{i}(t, \mathbf{r}) d^{3} r+\left\langle\frac{\partial \hat{H}_{r}}{\partial t}\right\rangle .
\end{aligned}
$$

Второе слагаемое в правой части (55) описывает взаимодействие с термостатами, а первое - действие механических сил. С учетом уравнения (28) оно имеет вид

$$
\begin{aligned}
& -\int_{V} \int_{V} \int_{0}^{\infty} \chi_{i j}\left(\tau, \mathbf{r}, \mathbf{r}^{\prime}, \mathbf{F}, \mathbf{B}, \mathbf{T}\right) \frac{d f_{i}(t, \mathbf{r})}{d t} \\
& \times \frac{f_{j}\left(t+\tau, \mathbf{r}^{\prime}\right)+f_{j}\left(t-\tau, \mathbf{r}^{\prime}\right)}{2} d \tau d^{3} r d^{3} r^{\prime} \\
& +\int_{V} \int_{V} \int_{0}^{\infty} \chi_{i j}\left(\tau, \mathbf{r}, \mathbf{r}^{\prime}, \mathbf{F}, \mathbf{B}, \mathbf{T}\right) \frac{d f_{i}(t, \mathbf{r})}{d t} \\
& \times \frac{f_{j}\left(t+\tau, \mathbf{r}^{\prime}\right)-f_{j}\left(t-\tau, \mathbf{r}^{\prime}\right)}{2} d \tau d^{3} r d^{3} r^{\prime} .
\end{aligned}
$$

Первое слагаемое в формуле (56) меняет знак при инверсии времени, т.е. описывает обратимый обмен энергии системы с источниками механических сил $\mathbf{F}$.
Ему можно сопоставить мощность работы $P_{F}$, совершаемой над системой источниками внешних сил. Второе слагаемое не меняет знак при инверсии времени, т.е. описывает необратимый обмен энергии системы с источниками сил F. Ему можно сопоставить тепловую мощность $Q_{F}$, создаваемую в системе под действием внешних механических сил. В квазистационарном режиме разложим в подынтегральном выражении формулы (56) силы в ряд Тейлора в окрестности точки $t$

$$
\begin{gathered}
Q_{F}(t)=\int_{V} \int_{V} \int_{0}^{\infty} \tau \chi_{i j}\left(\tau, \mathbf{r}, \mathbf{r}^{\prime}, \mathbf{F}, \mathbf{T}\right) \frac{\partial f_{i}(t, \mathbf{r})}{\partial t} \\
\times \frac{\partial f_{j}\left(\mathbf{r}^{\prime}\right)}{\partial t} d \tau d^{3} r d^{3} r^{\prime}, \\
P_{F}(t)=-\iint_{V} \int_{0}^{\infty} \chi_{i j}\left(\tau, \mathbf{r}, \mathbf{r}^{\prime}, \mathbf{F}, \mathbf{T}\right) \frac{\partial f_{i}(t, \mathbf{r})}{\partial t} \\
\times\left\{f_{j}\left(t, \mathbf{r}^{\prime}\right)+\frac{\tau^{2}}{2} \frac{\partial^{2} f_{j}\left(t, \mathbf{r}^{\prime}\right)}{\partial t^{2}}\right\} d \tau d^{3} r d^{3} r^{\prime} .
\end{gathered}
$$

В случае электрического воздействия полагаем в (55) $f(t, \mathbf{r})=\rho(t, \mathbf{r})$, используем соотношения (23) и интегрируем в первом уравнении по частям по переменным $r$ и $r^{\prime}$. Для плотности тепловой мощности с учетом формул (52) и (54) получим

$$
\begin{gathered}
q_{F}(t, \mathbf{r})=j_{\alpha}(t, \mathbf{r}) \int_{V} \lambda_{\alpha \beta}\left(\mathbf{r}, \mathbf{r}^{\prime}, \mathbf{j}, \mathbf{B}, \mathbf{T}\right) j_{\beta}\left(t, \mathbf{r}^{\prime}\right) d^{3} r^{\prime}, \\
\xi\left(\mathbf{r}, \mathbf{r}^{\prime}, \rho, \mathbf{B}, \mathbf{T}\right)=-\int_{0}^{\infty} \chi\left(\tau, \mathbf{r}, \mathbf{r}^{\prime}, \rho, \mathbf{B}, \mathbf{T}\right) d \tau, \\
p_{F}(t, \mathbf{r})=\frac{\partial \rho(t, \mathbf{r})}{\partial t} \int_{V} \xi\left(\mathbf{r}, \mathbf{r}^{\prime}, \rho, \mathbf{B}, \mathbf{T}\right) \rho\left(t, \mathbf{r}^{\prime}\right) d^{3} r^{\prime} \\
+\frac{j_{\alpha}(t, \mathbf{r})}{c} \int_{V} \xi_{\alpha \beta}\left(\mathbf{r}, \mathbf{r}^{\prime}, \mathbf{j}, \mathbf{A}, \mathbf{T}\right) \frac{\partial j_{\beta}\left(t, \mathbf{r}^{\prime}\right)}{\partial t} d^{3} r^{\prime} .
\end{gathered}
$$

С учетом уравнений (58) и (53) из уравнения (51) получаем

$$
\begin{aligned}
& \mathbf{E}(t, \mathbf{r}) \mathbf{j}(t, \mathbf{r})=q_{F}(t, \mathbf{r})+\mathbf{j}(t, \mathbf{r}) \frac{\partial}{\partial \mathbf{r}} \\
& \times \int_{V} \xi\left(\mathbf{r}, \mathbf{r}^{\prime}, \rho, \mathbf{B}, \mathbf{T}\right) \rho\left(t, \mathbf{r}^{\prime}\right) d^{3} r^{\prime}=q_{F}(t, \mathbf{r}) \\
& +\operatorname{div}\left\{\mathbf{j}(t, \mathbf{r}) \int_{V} \xi\left(\mathbf{r}, \mathbf{r}^{\prime}, \rho, \mathbf{B}, \mathbf{T}\right) \rho\left(t, \mathbf{r}^{\prime}\right) d^{3} r^{\prime}\right\} \\
& +\frac{\partial \rho(t, \mathbf{r})}{\partial t} \int_{V} \xi\left(\mathbf{r}, \mathbf{r}^{\prime} \rho, \mathbf{B}, \mathbf{T}\right) \rho\left(t, \mathbf{r}^{\prime}\right) d^{3} r^{\prime} .
\end{aligned}
$$


Если нелокальности не существенны, положим

$$
\begin{aligned}
& \xi\left(\mathbf{r}, \mathbf{r}^{\prime}, \rho, \mathbf{B}, \mathbf{T}\right)=\xi(\mathbf{r}, \rho, \mathbf{B}, \mathbf{T}) \delta\left(\mathbf{r}-\mathbf{r}^{\prime}\right), \\
& \lambda_{i j}\left(\mathbf{r}, \mathbf{r}^{\prime}, \mathbf{j}, \mathbf{B}, \mathbf{T}\right)=\lambda(\mathbf{r}, \mathbf{j}, \mathbf{B}, \mathbf{T}) \delta\left(\mathbf{r}-\mathbf{r}^{\prime}\right), \\
& \xi_{i j}\left(\mathbf{r}, \mathbf{r}^{\prime}, \mathbf{j}, \mathbf{A}, \mathbf{T}\right)=\xi(\mathbf{r}, \mathbf{j}, \mathbf{A}, \mathbf{T}) \delta\left(\mathbf{r}-\mathbf{r}^{\prime}\right) .
\end{aligned}
$$

При этом из уравнения (54) следует, что

$$
\begin{gathered}
\lambda_{\beta \alpha}(\mathbf{r},-\mathbf{j},-\mathbf{B}, \mathbf{T})=\lambda_{\alpha \beta}(\mathbf{r}, \mathbf{j}, \mathbf{B}, \mathbf{T}), \\
\xi_{\beta \alpha}(\mathbf{r}, \mathbf{j}, \mathbf{A}, \mathbf{T})=\xi_{\alpha \beta}(\mathbf{r}, \mathbf{j}, \mathbf{A}, \mathbf{T}) .
\end{gathered}
$$

Тогда первое уравнение (58) принимает вид закона Джоуля-Ленца для нелинейной анизотропной неоднородной среды без дисперсии

$$
\begin{gathered}
q_{F}(t, \mathbf{r}, \mathbf{j}, \mathbf{B}, \mathbf{T})=\lambda_{i j}(\mathbf{r}, \mathbf{j}, \mathbf{B}, \mathbf{T}) j_{i}(t, \mathbf{r}) j_{j}(t, \mathbf{r}), \\
q_{F}(t, \mathbf{r},-\mathbf{j},-\mathbf{B}, \mathbf{T})=q_{F}(t, \mathbf{r}, \mathbf{j}, \mathbf{B}, \mathbf{T})
\end{gathered}
$$

Второе уравнение (58) при этом принимает вид

$$
\begin{aligned}
& p_{F}(t, \mathbf{r})=\frac{\partial}{\partial t}\left\{\frac{\xi(\mathbf{r}, \rho, \mathbf{B}, \mathbf{T}) \rho^{2}(t, \mathbf{r})}{2}\right. \\
& \left.+\frac{\xi_{\alpha \beta}(\mathbf{r}, \mathbf{j}, \mathbf{A}, \mathbf{T}) j_{\alpha}(t, \mathbf{r}) j_{\beta}(t, \mathbf{r})}{2 c}\right\} .
\end{aligned}
$$

При анализе уравнения (56) мы считали, что первое слагаемое, т.е. мощность работы внешних сил $P_{F}$, описывает обратимый обмен энергии системы с источниками механических сил $\mathbf{F}$, т.е. взятую с обратным знаком максимальную работу, совершаемую системой над внешней средой при постоянной температуре. Поэтому выражение в фигурных скобках в правой части уравнения (61) является плотностью свободной энергии $\phi(t, \mathbf{r})$, такой, что функционал Масье-Планка во втором уравнении (11) имеет вид $\Phi(t)=\int_{V} \phi(t, \mathbf{r}) d^{3} r$. Первое слагаемое в фигурных скобках в правой части уравнения (61) можно рассматривать как электрическую составляющую плотности свободной энергии, создаваемую распределенными зарядами. Второе слагаемое, имеющее в соответствии с (53) вид $\mathbf{j}(t, \mathbf{r}) \mathbf{A}(t, \mathbf{r}) /(2 c)$, можно рассматривать как магнитную составляющую плотности свободной энергии, создаваемую распределенными токами.

Уравнение (59), в свою очередь, принимает вид

$$
\begin{aligned}
& \mathbf{E}(t, \mathbf{r}) \mathbf{j}(t, \mathbf{r})=q_{F}(t, \mathbf{r}, \mathbf{j}, \mathbf{B}, \mathbf{T})+\frac{\partial}{\partial t}\left\{\frac{\xi(\mathbf{r}, \rho, \mathbf{B}, \mathbf{T}) \rho^{2}(t, \mathbf{r})}{2}\right\} \\
& +\operatorname{div}\{\xi(\mathbf{r}, \rho, \mathbf{B}, \mathbf{T}) \rho(t, \mathbf{r}) \mathbf{j}(t, \mathbf{r})\}
\end{aligned}
$$

Левая часть уравнения (62) равна работе, совершаемой в единицу времени электрическим полем над зарядами в единице объема среды. Она расходуется на изменение электрической составляющей плотности свободной энергии и создание плотностей тепловой мощности и потока энергии. В электрически локально нейтральной системе $\mathbf{E}(t, \mathbf{r}) \mathbf{j}(t, \mathbf{r})=q_{F}(t, \mathbf{r}, \mathbf{j}, \mathbf{B}, \mathbf{T})$.

Если под действием механических сил в системе возникают ламинарные потоки компонент со скоростями $\mathbf{v}_{i}(t, r)=\left\langle\hat{\mathbf{j}}_{i}(t, r)\right\rangle /\left\langle\hat{c}_{i}(t, r)\right\rangle$, то плотность гамильтониана в локально квазиравновесном операторе плотности (11) преобразуется с учетом трансформационных свойств (24) [16]:

$$
\begin{aligned}
& \hat{\rho}^{q}=\exp \left\{-\Phi(t)-\int_{V} \theta(t, \mathbf{r})\left(\hat{h}_{0}(t, \mathbf{r})-\left(\mu_{i}(t, \mathbf{r})\right.\right.\right. \\
& \left.\left.\left.-m_{i} v_{i}^{2}(t, \mathbf{r})\right) \hat{c}_{i}(t, \mathbf{r})-\mathbf{v}(t, \mathbf{r}) \pi(t, \mathbf{r})\right) d^{3} r\right\},
\end{aligned}
$$

при этом функционал Масье-Планка $\Phi(t)$ в соответствии с (61) также зависит от механических сил. Из уравнения (11) следует, что эта зависимость обусловлена зависимостью от механических сил локальной температуры, например, из-за выделения тепла в системе и химических потенциалов компонент.

\section{Заключение}

В работе под нелинейной подразумевается система, отклик которой на внешнее воздействие не удовлетворяет принципу суперпозиции. В рамках предложенного подхода нелинейность системы возникает вследствие отклонения оператора плотности от локальноквазиравновесного из-за внешних воздействий. Такое отклонение возможно при внешнем воздействии, превышающем уровень, зависящий от структуры системы, например, от негармонических слагаемых в невозмущенном гамильтониане.

Кинетические коэффициенты (30) и (31) для отклика на механические и термодинамические воздействия получены по схеме Кубо [2] формально, без детализации физического механизма этого отклика. Вывод этих соотношений и основанного на них соотношения взаимности (34) справедлив в приближении марковской релаксации, если невозмущенный гамильтониан является стационарным, и невозмущенная система находится в квазиравновесном состоянии (11). Кроме того, система под действием возмущения, которое можно представить в виде суммы произведений классической заданной силы на оператор, соответствующей внешней динамической переменной и оператора марковской релаксации к квазиравновесному состоянию, остается устойчивой. Применимость марковской релаксации и локально квазиравновесного оператора рассмотрена соответственно в [14] и [15]. Здесь можно использовать принцип ослабления корреляций Боголюбова [19,20]. Для открытых диссипативных систем, взаимодействующих с термостатами, немарковские процессы релаксации затухают гораздо быстрее, чем основная марковская релаксация. Поэтому можно считать, что соотношение (30) и последующие справедливы при $\tau \geqslant \tau_{m}$, где $\tau_{m}$ - характерное время 
перехода системы к марковской релаксации, зависящее от структуры системы.

Преобразования кулоновского потенциала в (3) и далее представляют собой обычное мультипольное разложение и нужны, чтобы записать уравнение (10) в квазиклассической форме, когда внешние воздействия рассматриваются как классические поля, описываемые заданными функциями времени и координат, а динамические переменные - как квантовые величины, характеризующиеся эрмитовыми операторами. Сделанные при этом предположения необходимы именно для обоснования применимости квазиклассического представления и довольно сильно ограничивают применимость теории. Можно рассматривать внешние воздействия как операторные переменные и воспользоваться формализмом квантованных полей. Однако при этом матрица плотности открытой системы, взаимодействующей с квантованными полями, имеет сложную многоиндексную структуру, выкладки становятся очень громоздкими. Возможно, результат в замкнутой форме удастся получить с помощью формализма Крауса [21] и установить, являются ли полученные соотношения взаимности следствием квазиклассического рассмотрения, или они справедливы и для квантованных полей. Возможно, при этом удастся проанализировать и ограничения на характер релаксации.

Локальные температуры и химические потенциалы, входящие в уравнение (17), рассматриваются как заданные классические параметры. Разумеется, в реальных открытых системах, взаимодействующих с термостатамиисточниками частиц, они являются динамическими переменными, и им должны соответствовать эрмитовы операторы, зависящие от координат и времени. Для получения самосогласованных уравнений эти операторы нужно построить. Поскольку температура и химический потенциал - экстенсивные величины, их локальные операторы нельзя построить стандартным образом как операторы плотности некоторых аддитивных величин [16]. Для того чтобы связать их с оператором плотности энтропии, нужно построить его для неравновесной системы. В настоящее время эта задача еще не решена.

В последнее десятилетие взаимосвязь магнитных и электрических свойств вида (50) обнаружена при комнатной температуре в мультиферроиках, которые являются перспективными материалами для информационных и энергосберегающих технологий. Основные виды магнитоэлектрического взаимодействия, их механизмы и условия возникновения рассмотрены в [22]. Исследования [23-25] подтверждают эффективность управления динамикой намагниченности механическими напряжениями и электрическим полем. В работах [26,27] показано, что механическое напряжение служит „упругим калибровочным полем“, взаимодействующим с фермионами подобно электромагнитному полю. Исследованная в работе холловская вязкость описывается соотношением (30), когда в вектор $\mathbf{F}(t, \mathbf{r})$ включены компоненты магнитного поля и тензора напряжений, а в качестве отклика рассматриваются компоненты намагниченности и тензора деформаций. Величина этого калибровочного поля может быть охарактеризована соответствующим эффективным магнитным полем. В работе [28] теоретическая оценка составляет $10 \mathrm{~T}$, экспериментальные оценки по уровням Ландау показывают, что индуцированное механическими напряжениями поле может превышать 300 Т [29]. Такое поле может обеспечить эффективную поляризацию спинового тока при комнатной температуре.

Авторы исследования [30] на основе микроскопической теории показали, что температурный градиент в ферромагнетиках эквивалентен эффективному электрическому полю и порождает спиновой ток. Его зависимость от градиента температуры согласуется с соотношением (48). Возможность управления потоками тепла с помощью спинового тока подтверждена в работе [31]. Такое управление эффективно при высоких плотностях воздействий, когда система становится существенно нелинейной. Предложенный метод анализа квантовых транспортных эффектов, обусловленных поляризацией механически индуцированного спинового тока, создает основу для проектирования и оптимизации характеристик эффективных систем теплового транспорта.

\section{Конфликт интересов}

Авторы заявляют, что у них нет конфликта интересов.

\section{Список литературы}

[1] L. Onsager. Phys. Rev., 37, 405 (1931).

[2] R. Kubo. J. Phys. Soc. Jpn., 12 (6), 570 (1957). doi.org/ 10.1143/JPSJ.12.570

[3] R. Kubo, M. Yokota, S. Nakajima. J. Phys. Soc. Jpn., 12 (11), 1203 (1957). DOI: 10.1143/JPSJ.12.1203

[4] A. Hirohata, K. Yamada, Y. Nakatani, I.-L. Prejbeanu, B. Dieny, P. Pirro, B. Hillebrands. JMMM, 509, 166711 (2020). DOI: $10.1016 /$ j.jmmm.2020.166711

[5] А.А. Бухараев, А.К. Звездин., А.П. Пятаков, Ю.К. Фетисов. УФН, 188 (12), 1288 (2018). DOI: $10.3367 /$ UFNr.2018.01.038279

[A.A. Bukharaev, A.K. Zvezdin, A.P. Pyatakov, Y.K. Fetisov. Physics-Uspekhi, 61 (12), 1175 (2018). DOI: 10.3367/UFNe.2018.01.038279]

[6] G.E.W. Bauer, E. Saito, B.J. van Wees. Nature Mater., 11, 391 (2012). DOI: 10.1038/NMAT3301

[7] A. Miura, H. Sepehri-Amin, K. Masuda, H. Tscuchira, V. Miura, R. Iguchi, Y. Sakuraba, J. Shiomi, K. Hono, K. Uchida. Appl. Phys. Lett., 115, 222403 (2019). DOI: $10.1063 / 1.5131001$

[8] S. Ota, K. Uchida, R. Iguchi, P. Van Thach, H. Awano, D. Chibal. Scientif. Repor., 9, 13197 (2019). DOI: $10.1038 / \mathrm{s} 41598-019-49567-2$

[9] T. Hirai, H. Sepehri-Amin, K. Hasegavwa, T. Koyama, R. Iguchi, T. Ohkubo, D. Chiba, K. Uchida. Appl. Phys. Lett., 118, 022403 (2021). DOI: 10.1063/5.0034858

[10] F.K. Dejene, J. Flipse, B.J. van Wees. Phys. Rev. B, 90, 180402(R) (2014). DOI: 10.1103/PhysRevB.90.180402 
[11] В.К. Игнатьев. Письма в ЖТФ, 45 (11), 34 (2019). DOI: $10.21883 /$ JTF.2022.01.51861.126-21 [V.K. Ignat'ev. Tech. Phys. Lett., 45 (6), 563 (2019). DOI: $10.1134 / \mathrm{S} 1063785019060075]$

[12] В.К. Игнатьев, С.В. Перченко. ЖТФ, 87 (6), 837 (2017). DOI: $\quad 10.21883 / J T F .2017 .06 .44504 .1942$ [V.K. Ignatjev, S.V. Perchenko. Tech. Phys., 62 (6), 852 (2017). DOI: $10.1134 / \mathrm{S} 1063784217060135]$

[13] A. Rivas. Phys. Rev. Lett., 124 (16), 0601 (2020). DOI: 10.1103/PhysRevLett.124.160601

[14] В.Ю. Шишков, Е.С. Андрианов, А.А. Пухов, А.П. Виноградов, А.А. Лисянский. УФН, 189 (5), 544 (2019). DOI:10.3367/UFNr.2018.06.038359

[V.Yu. Shishkov, E.S. Andrianov, A.A. Pukhov, A.P. Vinogradov, A.A. Lisyansky. Physics-Uspekhi, 62 (5), 510 (2019). DOI: $10.3367 /$ UFNe.2018.06.038359]

[15] Д.Н. Зубарев, В.Г. Морозов, Г. Репке. Статистическал механика неравновесных процессов (Физматлит, М., 2002), т. 1. [D. Zubarev, V. Morozov, G. Ropke. Statistical Mechanics of Nonequilibrium Processes (Akademie Verlag, 1997), v. 1.]

[16] А.И. Ахиезер, С.В. Пелетминский. Методы статистической физики (Наука, М., 1977). [A.I. Akhieser, S.V. Peletminsky. Methods of Statistical Physics (Nauka, M., 1977)]

[17] М.А. Леонтович. Введение в термодинамику, статистическая физика (Наука, М., 1983) [M.A. Leontovich. An Introduction to Thermodynamics, Statistical Physics (Nauka, M., 1983)]

[18] С.Р. де Гроот, П. Мазур. Неравновесная термодинамика (Мир, М., 1964) [S.R. de Groot, P. Mazur. NonEquilibrium Thermodynamics (North-Holland Publishing Company, Amsterdam, 1962).]

[19] Н.Н. Боголюбов. Избранные труды в трех томах (Наукова думка, Киев, 1971), т. 3, с. 166-173. [N.N. Bogolubov. Selected works in 3 volumes. (Scientific Thought, Kiev, 1971), v. 3, p. 166-173.]

[20] Н.Н. Боголюбов. Избранные университетские лекции (МГУ, М., 2009), c. 338-352. [N.N. Bogolubov. Selected University Lectures (Moscow University Press, M., 2009), p. 338-352.]

[21] K. Kraus. States, Effects, and Operations: Fundamental Notions of Quantum Theory, ser. Lecture Notes in Physic (Springer-Verlag, 1983), v. 190. Lectures in Mathematical Physics at the University of Texas at Austin. DOI: $10.1007 / 3-540-12732-1$

[22] А.П. Пятаков, А.К. Звездин. УФН, 182 (6), 593 (2012). DOI: $10.3367 /$ UFNr.0182.201206b.0593 [A.P. Pyatakov, A.K. Zvezdin. Physics-Uspekhi, 55 (6), 557 (2012). DOI: $10.3367 /$ UFNr.0182.201206b.0593]

[23] K. Roy, S. Bandyopadhyay, J. Atulasimha. Phys. Rev. B, 83 (22), 4412 (2011). DOI: 10.1103/PhysRevB.83.224412

[24] C.-Y. Liang, A. Sepulveda, S. Keller, G.P. Carman. J. Appl. Phys., 119 (11), 3903 (2016). DOI: http://dx.doi.org/10.1063/1.4944650

[25] A. Khan, D. Nikonov, S. Manipatruni, T. Ghani, I.A. Young. Appl. Phys. Lett., 104 (26), 2407 (2014). DOI: http://dx.doi.org/10.1063/1.4884419

[26] A. Cortijo, Y. Ferreirós, K. Landsteiner, M.A.H. Vozmediano. 2D Mater, 3 (1), 1002 (2016). DOI: $10.1088 / 2053-1583 / 3 / 1 / 011002$
[27] V.K. Ignatiev, N.G. Lebedev, A.A. Orlov, S.V. Perchenko. JMMM, 494, 165658 (2020) DOI: $10.1016 /$ j.jmmm.2019.165658

[28] F. Guinea, M.I. Katsnelson, A.K. Geim. Nature Phys., 6, 30 (2010). DOI: $10.1038 /$ NPHYS1420

[29] N. Levy, S.A. Burke, K.L. Meaker, M. Panlasigui, A. Zettl, F. Guinea, A.H. Castro Neto, M.F. Crommie. Science, 329, 544 (2010). DOI: 10.1126/science. 1191700

[30] Y. Takezoe, K. Hosono, A. Takeuchi, G. Tatara. Phys. Rev. B, 82 (9), 094451 (2010). DOI: 10.1103/PhysRevB.82.094451

[31] I.A. Starkov, O.V. Pakhomov, A.S. Starkov. JMMM, 496, 165949 (2020). DOI: 10.1016/j.jmmm.2019.165949 